Preprint PUPT-1713, MCGILL-97/24, DUKE-TH-97-154 e-Print Archive: hep-ph/9710436

\title{
Chern-Simons number diffusion with hard thermal loops
}

\author{
Guy D. Moore \\ Dept. of Physics, McGill University \\ 3600 University St. \\ Montreal, PQ H3A 2T8 Canada \\ Chaoran Hu and Berndt Müller \\ Department of Physics, Duke University \\ Durham, North Carolina 27708-0305, USA
}

\begin{abstract}
We construct an extension of the standard Kogut-Susskind lattice model for classical 3+1 dimensional Yang-Mills theory, in which "classical particle" degrees of freedom are added. We argue that this will correctly reproduce the "hard thermal loop" effects of hard degrees of freedom, while giving a local implementation which is numerically tractable. We prove that the extended system is Hamiltonian and has the same thermodynamics as dimensionally reduced hot Yang-Mills theory put on a lattice. We present a numerical update algorithm and study the abelian theory to verify that the classical gauge theory self-energy is correctly modified. Then we use the extended system to study the diffusion constant for Chern-Simons number. We verify the Arnold-Son-Yaffe picture that the diffusion constant is inversely proportional to hard thermal loop strength. Our numbers correspond to a diffusion constant of $\Gamma=29 \pm 6 \alpha_{\mathrm{w}}^{5} T^{4}$ for $m_{\mathrm{D}}^{2}=11 g^{2} T^{2} / 6$.
\end{abstract}

PACS numbers: 11.10.Wx, 11.15.Ha, 11.15.Kc

\section{Introduction}

One of the most profound and most poorly explained observations of modern cosmology is that the universe contains macroscopic amounts of matter, but not of antimatter. Since baryon number is conserved in all observed terrestrial and astrophysical phenomena, the existence of such a baryon number asymmetry (baryons over antibaryons) seems peculiar, particularly because it clearly violates $C$ and $C P$ symmetry. Further, the abundance of baryons, as compared to the abundance of photons or the entropy density of the universe, is a remarkably small number, on order $10^{-10}$; and since the entropy of the universe has changed very little since its very hot early epochs, this small nonzero number is an initial condition to the universe viewed at least back to the epoch of primordial nucleosynthesis.

Sakharov [1] made the first attempt to understand what is involved in explaining this observation. If the universe does not begin with such an asymmetry, then baryon number must be violated to generate it; so must $C$ and $C P$. He also noted that if baryon number 
is not conserved, then in equilibrium it will go to zero, as a consequence of $C P T$ symmetry. Hence, if the baryon asymmetry of the universe was generated dynamically, the universe must in its early history have gone through some departure from thermal equilibrium, in which baryon number violation was active and $C$ and $C P$ violating physics were relevant, followed by a steep suppression of the rate of baryon number violation before thermal equilibrium resumed (to prevent the baryon number from being erased again). And if baryon number was violated at a rate faster than the Hubble expansion at any point in the universe's history, then the baryon number must have been generated dynamically.

While it is known that grand unified theories (GUTs) generically violate baryon number, it turns out that baryon number is violated already in the standard model, as first shown by t'Hooft [2]. The key observation is that baryon number is coupled through the axial anomaly to the Chern-Simons number $\left(N_{\mathrm{CS}}\right)$ of the $\mathrm{SU}(2)$ weak hypercharge field,

$$
\frac{d N_{\mathrm{B}}}{d t}=N_{\mathrm{F}} \frac{d N_{\mathrm{CS}}}{d t}, \quad\left(N_{\mathrm{F}}=3\right)
$$

and that $N_{\mathrm{CS}}$ change is thermally activated in the hot electroweak plasma [3, 4, 5]. This baryon number violation occurs at much lower temperature ranges, and hence later in the evolution of the early universe, than GUT mechanisms; it could therefore erase any baryon

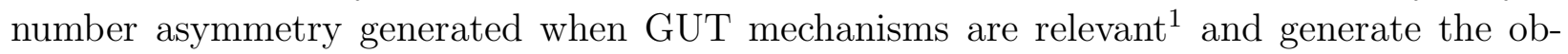
served abundance during the electroweak phase transition, when the rate abruptly shuts off. This latter possibility has stimulated the field of electroweak baryogenesis.

One thing we need to know to understand electroweak baryogenesis is how quickly baryon number is violated in the standard model, above the electroweak phase transition. The violation involves nonperturbative physics, and there are no known analytic methods which are reliable above the phase transition temperature (in contrast to the situation below it [3], 5]). Already at the thermodynamic level the fields responsible for the violation of baryon number suffer from the "infrared problem" of thermal field theories with light interacting bosons.

The "infrared problem" of the thermodynamics of thermal field theories with light interacting bosons can be "solved" by the dimensional reduction procedure [6, 7, 8], which reduces the problem to a three dimensional path integral which can be put on a lattice [9, 10, 11] and studied numerically [12, 13, 14, 15, 16]. This "dimensional reduction" turns out to be identical to considering the partition function of the $3+1$ dimensional, classical bosonic theory, with certain counterterms [17]. The most hopeful approach to the study of infrared sensitive, dynamical properties of the plasma is that they also behave essentially classically [18], and there is reason to believe that they do [15, 19.

There is a problem with the classical approximation to the dynamics, however. The ultraviolet modes $(p \sim \pi T)$ certainly do not behave as classical fields, and they interact with the infrared modes (which we will assume do behave essentially classically). The classical approximation will serve only if the interaction between soft and hard modes is somehow unimportant to the evolution of the soft modes. It is known that, at weak coupling, the hard modes only influence the thermodynamics of the soft modes by shifting the Higgs mass

\footnotetext{
${ }^{1}$ The standard model violates $B+L$ but not $B-L$. Extended GUTs such as $\mathrm{SO}(10)$ can produce a $B-L$ excess, which electroweak processes will not touch. But the possibility of $B$ production in the standard model certainly motivates the study of these scenarios.
} 
and the Debye screening mass for the $A_{0}$ field. However, the unequal time generalization of Debye screening, the hard thermal loops, constitute very nontrivial physics, physics which the lattice implementation of the classical theory gets wrong [20], both in the size of the screening effects (which depend inversely on the lattice spacing) and in the functional details.

Does this problem matter to the rate at which baryon number is violated? In particular, since there is a fluctuation dissipation relation which relates the rate at which a baryon number excess is dissipated in the plasma to the rate of $N_{\mathrm{CS}}$ diffusion per unit volume[21], we might ask whether the hard thermal loops matter to the $N_{\mathrm{CS}}$ diffusion constant. The numerical lattice results of Ambjørn and Krasnitz implied that there is a well defined small lattice spacing limit to the classical, lattice diffusion constant [17], but the definition of lattice $N_{\text {CS }}$ used there gives bizarre results in the broken phase [22] due to serious lattice artifacts [15]. Theoretical arguments suggest that hard thermal loops should slow down the evolution of infrared magnetic fields, for the same reason that infrared magnetic fields in an abelian plasma become pinned by the conductivity of the plasma [23, 24]; hence one should expect that the $N_{\mathrm{CS}}$ diffusion constant vanishes linearly with lattice spacing in classical simulations. Recently, numerical techniques based on a topological definition of $N_{\mathrm{CS}}$ 25] and a "cooled field" definition which removes the worst problems of the old definition [26] have indicated a lattice spacing dependence in the diffusion constant, though it does not appear to be as large as expected. Hence, at present the situation seems confused, and it certainly seems necessary to find a way to include more faithfully the effects of hard thermal loops in the study of $N_{\mathrm{CS}}$ diffusion, and in other studies of infrared bosonic field evolution, such as the study of the phase transition dynamics [15] and the generation of quark gluon plasma in heavy ion collisions [27, 28, 29].

In this paper we develop, implement, and study a proposal by two of us [30] for including the hard thermal loop effects into classical, lattice simulations of Yang-Mills or Yang-Mills Higgs theory. The implementation produces a local, Hamiltonian system in which added "particle" degrees of freedom convey the hard thermal loop effects. We explicitly verify that the enlarged system conserves energy and symplectic measure, has the same thermodynamics as the quantum theory in the dimensional reduction approximation, and produces the right dynamical behavior for infrared fields. We examine in detail the retarded propagator of the abelian theory with particles to show that they correctly produce all of the physics of hard thermal loops, including Debye screening, plasma oscillations, and Landau damping. Then we apply the particle method to determine the $N_{\mathrm{CS}}$ diffusion constant and its dependence on the Debye mass. The results verify the arguments of Arnold, Son, and Yaffe (ASY). In particular, we verify that as the total strength of hard thermal loop effects varies by a factor of 3, with the lattice spacing and the physical volume held constant, the diffusion constant for $N_{\mathrm{CS}}$ also varies by a factor of 3 .

The outline of the paper is as follows. In Section 2 we review what the hard thermal loops are and how they can be understood in terms of Vlasov equations, ie in terms of the influence of a bath of particles on the classical infrared modes. In section 3 we explicitly construct a spatial lattice, continuum time system which can be viewed as an $N$ body simulation of the Vlasov equations. We show that this system is energy conserving and Hamiltonian and argue

\footnotetext{
${ }^{2}$ There are also perturbatively computable corrections to the couplings of the theory, which amount to a specification of the renormalization point, see Farakos et. al. [7. 8]
} 
that, for small charge and large particle number, it reduces to the (lattice) Vlasov equations in the eikonal approximation, which are known to produce the right hard thermal loops. In Section 6 we study its thermodynamics, which are shown to be the same as dimensionally reduced Yang-Mills theory put on the lattice, at a specific Debye mass set by the number and charge of the particles. Section 5 develops a stable, time symmetric update algorithm, and Section 6 numerically studies the retarded propagator of the abelian theory. Section 0 uses the evolution algorithm, together with the topological definition of $N_{\mathrm{CS}}$ developed in [25], to study the $N_{\mathrm{CS}}$ diffusion constant and its dependence on lattice spacing and on the size of hard thermal loop effects. Section 8 concludes. There are also four technical appendices. Appendix A analytically studies how the lattice nature of the electric fields changes the plasma frequency, in the limit of many particles of small charge. Appendix B studies the thermodynamics of the lattice system when $m_{\mathrm{D}} \sim g^{2} T / a$. Appendix $\mathrm{C}$ reviews how to probe the retarded propagator via linear response to an external current. Appendix $\mathrm{D}$ discusses the relation between lattice and continuum time scales.

\section{Hard thermal loops and particles}

In this section we briefly review an approach to hard thermal loops due to Kelly et al [31] and discuss how one can generate the influence of hard thermal loops by coupling the infrared fields to a heat bath of particles.

In the diagrammatic approach to field theory at high temperature it was shown by Braaten and Pisarski [32 that a resummation procedure is needed in order to take into account consistently all contributions at leading order in the coupling constant. Such contributions are called "hard thermal loops" since they arise from one-loop diagrams with soft external legs and hard internal momenta. The hard thermal loop resummation was shown to produce gauge invariant results for physical quantities such as the gluon damping rate in a QCD plasma [33]. An effective action for the hard thermal loops was derived by Taylor and Wong [34 by imposing gauge invariance on the generating functional. Another approach developed by Blaizot and Iancu [35] is based on a truncation of the hierarchy of SchwingerDyson equations and the generating functional was obtained through a consistent expansion in the coupling constant.

While remarkably insightful, the approaches mentioned above are quite technical, often involving lengthy calculations, and they tend to hide the classical nature of hard thermal loops. The hard thermal loops arise from loop diagrams and can be obtained from the Schwinger-Dyson equations of quantum field theory. But they are also UV finite, with loop integrals being exponentially suppressed in the ultraviolet. This is because they arise entirely from thermal fluctuations. One usually thinks of such fluctuations, at least in the ultraviolet, as being well described by classical particles, which motivated Kelly et al [31] to find an alternative, classical formalism for hard thermal loops, by considering the linear response of an ensemble of thermal particles to a weakly coupled, slowly varying mean field in the framework of classical transport theory [36]. They start by considering particles carrying non-abelian $\mathrm{SU}(N)$ charge $q^{a}, a=1, \ldots, N^{2}-1$. The Wong equations [37] are used to describe 
the proper time evolution of a particle with phase space coordinates $\left(\xi^{\mu}, p^{\mu}, q^{a}\right)$ :

$$
\begin{aligned}
m \frac{d \xi^{\mu}}{d \tau} & =p^{\mu}, \\
m \frac{d p^{\mu}}{d \tau} & =g q^{a} F_{a}^{\mu \nu} p_{\nu}, \\
m \frac{d q^{a}}{d \tau} & =-g f^{a b c} p^{\mu} A_{\mu}^{b} q^{c},
\end{aligned}
$$

where $g$ is the coupling constant and $F_{a}^{\mu \nu}$ denotes the strength of the mean color field $A_{a}^{\mu}$. Note that the color charge is itself subject to dynamical evolution, a feature which is absent in the abelian case. Both the dynamical evolution of a particle's spin and the spin coupling are neglected since spin interactions are down by an order of $g$ in a weak mean field describing soft excitations $\left(k \sim g T, A_{\mu} \lesssim T, F_{\mu \nu} \lesssim g T^{2}\right)$ [38].

Consider the classical one particle distribution function $f(x, p, q)$ evolving in time according to the Boltzmann transport equation,

$$
m \frac{d f(x, p, q)}{d \tau}=0
$$

where the collision integral describing hard collisions between particles is neglected since soft collisions mediated by the mean field dominate. Inserting the Wong equations (2)-(国) into (5), one arrives at the following:

$$
p^{\mu}\left[\frac{\partial}{\partial x^{\mu}}-g q^{a} F_{\mu \nu}^{a} \frac{\partial}{\partial p_{\nu}}-g f_{a b c} A_{\mu}^{b} q^{c} \frac{\partial}{\partial q^{a}}\right] f(x, p, q)=0 .
$$

A self-consistent set of non-abelian Vlasov equations for the distribution function and the mean color field can be obtained by augmenting (6) with the Yang-Mills equations,

$$
D_{\mu} F^{\mu \nu}=g \int d p d q p^{\nu} q f(x, p, q) \equiv j^{\nu}(x)
$$

The non-abelian Vlasov equations (6) and (7) are now applied to study the soft excitations in a hot, color-neutral plasma with massless particles. In the spirit of linear response theory, one expands the distribution function in powers of $g$,

$$
f=f^{(0)}+g f^{(1)}+\mathcal{O}\left(g^{2}\right)
$$

where $f^{(0)}\left(p_{0}\right)=C n_{\mathrm{B}, \mathrm{F}}\left(p_{0}\right)$ is the equilibrium distribution in the absence of a net color field. At leading order in $g$, the equation (6) reduces to

$$
p^{\mu}\left[\frac{\partial}{\partial x^{\mu}}-g f_{a b c} A_{\mu}^{b} q^{c} \frac{\partial}{\partial q^{a}}\right] f^{(1)}(x, p, q)=p^{\mu} q^{a} F_{\mu \nu}^{a} \frac{\partial}{\partial p_{\nu}} f^{(0)}\left(p_{0}\right) .
$$

Similarly, there is a net induced current density in momentum space,

$$
j^{\mu a}(x, p)=g^{2} \int d q p^{\mu} q^{a} f^{(1)}(x, p, q)
$$


From (9) and (10), a constraint satisfied by the color current can be derived. Using the standard field theory definition of effective action $j^{\mu}(x)=-\frac{\delta \Gamma(A)}{\delta A_{\mu}(x)}$, one then arrives at the hard thermal loop effective action of the following form:

$$
\Gamma_{\mathrm{HTL}}=\frac{1}{2} m_{\mathrm{D}}^{2}\left[\int d^{4} x A_{0}^{a}(x) A_{0}^{a}(x)-\int \frac{d \Omega}{(2 \pi)^{4}} W(A \cdot v)\right],
$$

where $v \equiv\left(1, \boldsymbol{p} / p_{0}\right), m_{\mathrm{D}}=g T \sqrt{\frac{2 N+N_{\mathrm{s}}+2 N_{\mathrm{F}}}{6}}$. 3 and the integration $\int d \Omega$ is over all directions of the unit vector $\boldsymbol{p} / p_{0}$. The first term describes Debye screening. $W(A \cdot v)$ in the second term is a functional. Its explicit form has been given by Taylor and Wong [34, also by Efraty and Nair [39]. Note that the derivation of $\Gamma_{\text {HTL }}$ stays completely within the classical transport theory and makes no use of the quantum theory. This justifies the statement that hard thermal loop effects are classical (in the sense of the classical particle approximation). Also note that the form of the expression inside the brackets in Eq. (11) does not rely on the particles obeying any specific statistics; they can be Fermi, Bose, or even Boltzmann particles. Nor does it depend on what group representation they are in. These only affect how much a species contributes to the leading coefficient $m_{\mathrm{D}}^{2}$, which is given by a sum over charged species of their individual contributions. The ratio of a particle species' contribution to $m_{\mathrm{D}}^{2}$ and the mean density of those particles is $2 g^{2} C_{2}(R)\left\langle E^{-1}\right\rangle$. (For classical, distinguishable particles in the adjoint representation, $g^{2} C_{2}(R)$ is replaced by the mean squared value of the particle charge $q$ in one Lie algebra direction.)

It is worth noting that the magnetic field does not play a role in producing hard thermal loops at leading order. This can be easily seen from (9), where only the electric field enters the term on the right. Physically, this is because the magnetic field just rotates the momentum distribution, and at leading order that distribution is the rotationally invariant thermal distribution. The magnetic field only influences existing departures from equilibrium, which is a subleading effect. In contrast, the electric field can polarize the plasma and generate a net current, which in turn interacts with the mean field and hence generates the desired hard thermal loops. Therefore, in solving the Wong equations, one can leave out the magnetic term in the Lorentz force in (3) if the plasma is only slightly driven out of equilibrium. However, if one is interested in dynamical processes occurring in out-of-equilibrium plasma, then the force due to the magnetic field has to be included.

The effective action (11) is conceptually simple and formally appealing. It provides a concise way of summarizing hard thermal loops and allows one to better understand the influence of the hard thermal modes on the soft excitations. Nevertheless, it does not prove a ready starting point for practical calculations. In particular, its nonlocality makes it hard to apply to study nonperturbative physics such as Chern-Simons number diffusion, where analytical methods are rare and one has to rely on numerical simulations. However, the fact that the hard thermal loop effective action (11) can be derived from classical transport theory, i.e. the Vlasov equations, implies that hard thermal loops can be generated by solving the Vlasov equations numerically. One could do so by an $N$ body simulation by solving the coupled system of Wong equations (国)-(田) and Yang-Mills equations (7). This

\footnotetext{
${ }^{3}$ Here, $N_{\mathrm{s}}$ is the number of fundamental scalars, and as in the preceding text, $N_{\mathrm{F}}$ is the number of generations, each containing four chiral doublets; in QCD $2 N_{\mathrm{F}}$ would be replaced by $N_{\mathrm{F}}$ the number of flavors, and $N_{\mathrm{s}}$ would only appear in supersymmetric extensions.
} 
has the advantage of being local in spacetime and allows a practical, real time study of both equilibrium and nonequilibrium phenomena.

Based on these ideas, our goal will be to derive a system of dynamical equations which, in its low-frequency, long-wavelength limit, reduces to the same effective field theory as the full thermal gauge theory. We have no intention of modeling the gauge theory as truly as possible in all respects; rather, we will be satified to reproduce its infrared behavior correctly. For example, it will be irrelevant for us whether the system of classical particles carrying gauge charge has detailed resemblance to the hard thermal modes of the gauge field, if only its back-reaction produces the nonlocal HTL effective action (11). The system of classical dynamical equations to be derived in the following sections will have three parameters: the classical thermal length scale $\left(g^{2} T\right)^{-1}$, the gauge charge $(g Q)^{2}$ of the particles, and the particle density $\langle n\rangle$. It will be sufficient for our purpose if we tune these parameters so that they produce the correct values for the parameters in the HTL action.

\section{The lattice system}

To reproduce the hard thermal loop effects, our idea is then to numerically implement "particles" obeying Wong's equations and moving in the background of classical, lattice fields. Eventually we want a discrete time update algorithm, but an important step is to construct a continuous time, lattice system with the right properties. The general philosophy of adding particles with adjoint (Lie algebra) charge $q$ of fixed magnitude, kinetic momentum $\vec{p}$, and continuous position coordinate $\xi$ satisfying $\dot{\xi}_{i}=p_{i} /|p|$, has been presented in [30]; here we will specify the complete implementation. Our requirements for this system are

- There must be conservation of energy,

- The evolution should preserve the phase space (symplectic) measure,

- The system should respect cubic, (lattice) translation, $C, P$, and $T$ symmetry,

- Gauss' law should be identically preserved,

- The small lattice spacing a limit (or smooth field, large time limit if one thinks in lattice units) must recover the Yang-Mills field equations supplemented with Wong's equations,

- The thermodynamics of the infrared classical fields must be given by the path integral of quantum Yang-Mills theory in the dimensional reduction approximation (or its lattice discretization).

Note that the first two conditions ensure that the system is Hamiltonian, and hence that the thermodynamics are well defined. We would also like a thermalization algorithm for the system.

We emphasize that the particles are a device to reproduce the hard thermal loops and should not be taken literally as reproducing all the behaviors of the hard modes. In particular it is not a problem that they are distinguishable, and that their number is conserved. We expect them to satisfy Boltzmann statistics, rather than Bose or Fermi-Dirac statistics, but 
this is also not important to reproducing the functional form of the hard thermal loops, as discussed in the last section; we need only make sure that the number density and charge of the particles yields the desired value for $m_{\mathrm{D}}^{2}$. However, any hope that the method can be enhanced to account for physics beyond hard thermal loops is clearly remote. Hence we will not be able to say anything about effects which are subleading in $g$.

Let us list the degrees of freedom of the proposed system (see Fig. 1 for illustration). We consider a 3-torus of spatial extent $N^{3}$ (in lattice units, which will be used throughout except when it is convenient to write the lattice spacing explicitly). "The lattice" will refer to the integer lattice on this space, ie all points with all three coordinates an integer. A link $x, i$ will refer to the line between the lattice site $x$ and the site $x+\hat{i}$ (henceforward $x+i$ ) and on each link there will be a parallel transporter $U_{i}(x) \in \mathrm{SU}(2)$ and an electric field $E_{i}(x) \in$ $\operatorname{LSU}(2)$, the Lie algebra of $\mathrm{SU}(2)$. For each index value $\alpha \in\left\{1, . ., N_{\mathrm{p}}\right\}$ there is a particle with coordinate $\xi_{\alpha}$ defined on the torus, momentum $p_{\alpha} \in \Re^{3}$, and charge $q_{\alpha} \in \operatorname{LSU}(2)$ satisfying $q_{\alpha}^{2}=Q^{2}$. By definition $E_{i}(x)$ will be the left acting covariant time derivative of $U_{i}(x), D_{0} U_{i}(x)=E_{i}^{a}(x) i \tau^{a} U_{i}(x)$, and the momentum $p_{\alpha}$ will tell the direction the particle moves in, $\dot{\xi}_{\alpha, i}=p_{\alpha, i} /|p|_{\alpha}$ (note the $\xi_{i}$ are defined $\bmod N$ ). It remains to define update rules for $E, p$, and $q$.

First, what is the meaning of $q$ ? It should be a charge which the classical fields $U, E$ "see," but such a charge should reside at a lattice point. We take $q_{\alpha}$ to "live" at the lattice point $x_{i}$ closest to $\xi_{\alpha}$, ie $\left|x_{i}-\xi_{\alpha, i}\right| \leq 0.5$. It will gauge transform as an adjoint object at that site; and the charge observed by the classical fields will be

$$
\rho(x)=\sum_{\alpha} q_{\alpha} \times\left\{\begin{array}{ll}
1 & \xi \text { at } x \\
0 & \xi \text { not at } x
\end{array} .\right.
$$

This is the quantity which enters Gauss' law,

$$
\rho(x)=\sum_{i} E_{i}(x)-U_{i}^{\dagger}(x-i) E_{i}(x-i) U_{i}(x-i) \equiv D_{\mathrm{L}} \cdot E(x) .
$$

The current $j_{i}^{a}$, an adjoint vector field, should be defined on the links and is specified by the requirement that it form a conserved current,

$$
\dot{\rho}=\sum_{i} U_{i}^{\dagger}(x-i) j_{i}(x-i) U_{i}(x-i)-j_{i}(x)
$$

The easiest way to see how this fixes the current is to think of the charge $q_{\alpha}$ of particle $\alpha$ as living at site $x$ until the particle moves to be closest to another site, say $x+i$; then the charge must abruptly slide along the link connecting $x$ and $x+i$, and there will be a $(\delta$ function in time) current on that link, equal to $j_{i}(x)=q_{\alpha} \delta\left(t-t_{\text {cross }}\right)$. If we take the group indicies of $j$ to live at the basepoint of the link $x$, then we should use the value of $q$ which departs from site $x$; the value if we take $j$ to live at the endpoint of the link $x+i$ is the adjoint parallel transport of $j$ with group indicies at the basepoint, and hence the charge $q$ of the particle when it arrives at site $x+i$ must be the parallel transport of the value at $x$,

$$
q(\text { at } x+i)=U_{i}^{\dagger}(x) q(\text { at } x) U_{i}(x) .
$$

If the particle is moving the other direction, from $x+i$ to $x$, the sign of the current is reversed, but (15) still holds. This gives the update rule for $q$ and is the same as was proposed in [30]. 


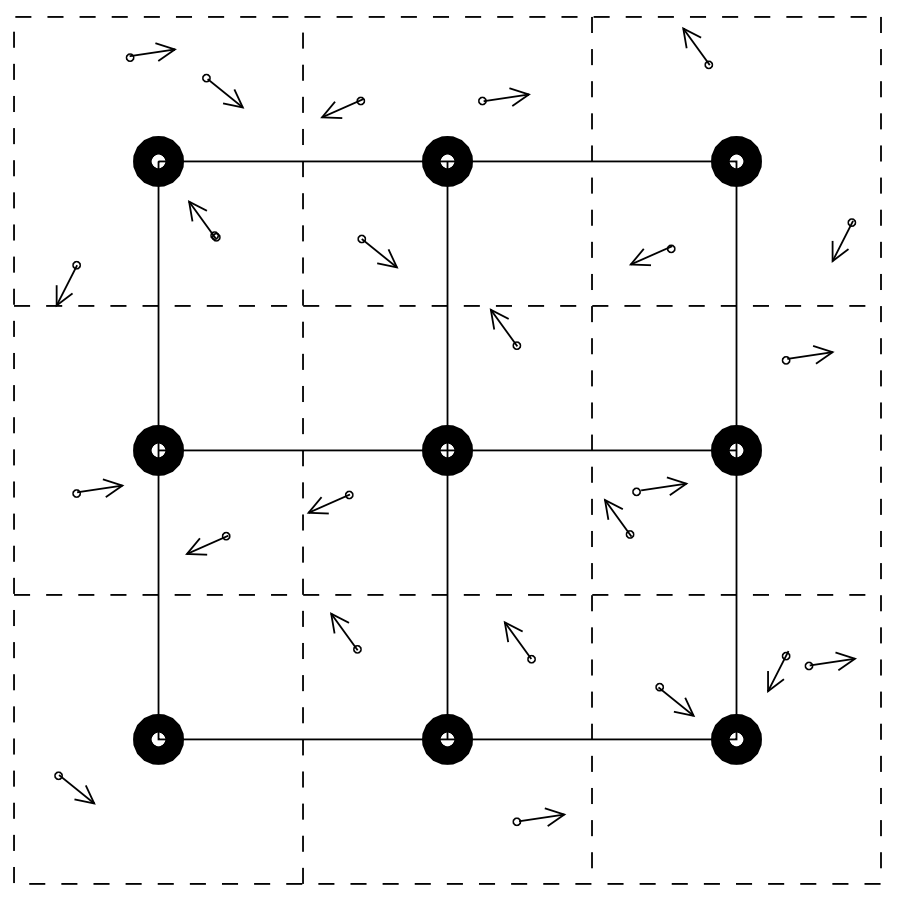

Figure 1: An illustration of the degrees of freedom of the proposed system. Lattice sites are large dots, and the solid lines joining them are links. Classical fields take values at sites (Higgs fields) or on links (connections $U$ and electric fields $E$ ). Particles (the small dots) take on real valued coordinates and momenta (illustrated with arrows). A particle's charge affects the classical fields as if it resided at the nearest lattice site. The dotted lines (really planes, extending out of the page) are barriers between the region nearest one site and that nearest another, that is, faces of the dual lattice. When a particle crosses a barrier, the charge is parallel transported to the new box, the $E$ field on the link orthogonal to the barrier receives a kick, and the particle momentum orthogonal to the barrier is changed to conserve energy.

In what follows we will call such events "boundary crossings" because they correspond to a particle crossing the boundary which demarks the volume closest to one lattice site. The boundary is a face of the dual lattice, dual to the link in which the current flows.

We expect that the electric field update rule should be

$$
\begin{aligned}
\frac{d E_{i}(x)}{d t} & =-\frac{\partial H_{\mathrm{KS}}}{\partial U_{i}(x)}-j_{i}(x) \\
H_{\mathrm{KS}} & \equiv \sum_{x, i} \frac{E_{i}^{2}(x)}{2}+\sum_{\square}\left(1-\frac{1}{2} \operatorname{Tr} U_{\square}\right)
\end{aligned}
$$

where $H_{\mathrm{KS}}$ is the Kogut-Susskind Hamiltonian [40] which is standard in the real time field literature [41], and where $\partial / \partial U_{i}(x)$ means change with respect to left acting derivatives of $U_{i}(x)$. The appearance of $j$ here is the "meaning" of $j$ and just means that $E_{i}(x)$ changes abruptly by $-q_{\alpha}$ when particle $\alpha$ moves from nearest site $x$ to nearest site $x+i$, and by $q_{\alpha}$ if it goes the other way. 

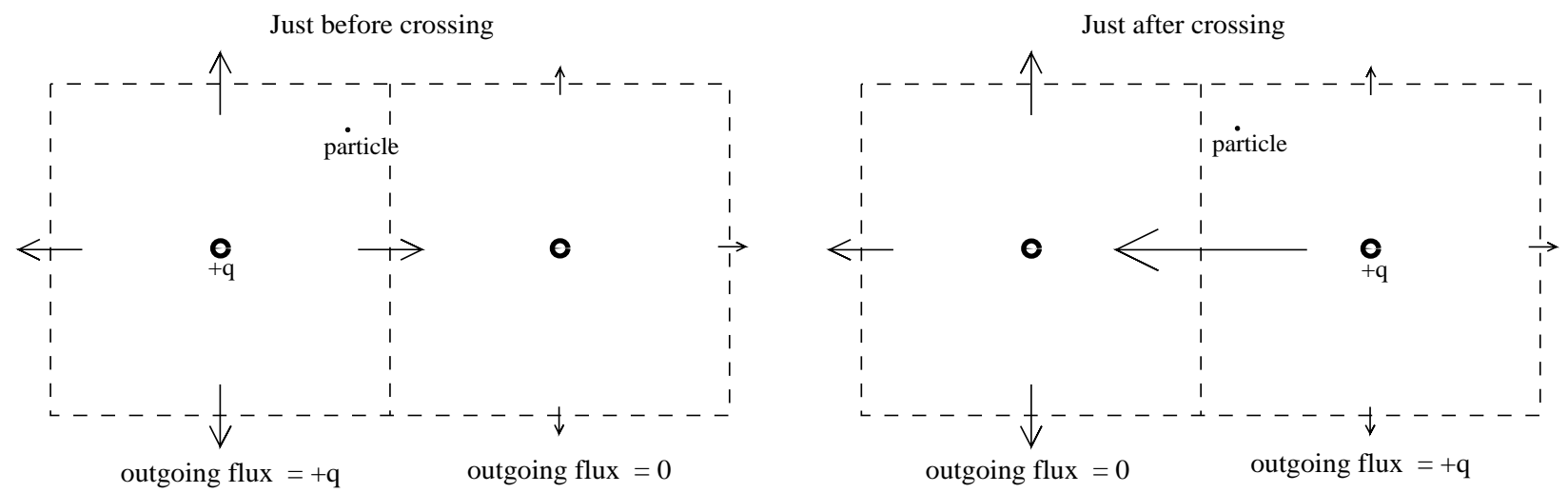

Figure 2: Boundary crossing, before and after. Before, the flux of $E$ fields (displayed as arrows) out of the lefthand box must equal $q$, and the flux from the righthand box must equal 0 . After the crossing, the righthand box has flux $q$ and the lefthand box has zero. This demands a change of the $E$ field connecting them, but no instantaneous change to the $E$ fields which leave these two boxes, since that would change the flux going into other boxes.

Now let us check whether this definition of $\dot{E}$ will preserve Gauss' law, that is, whether

$$
\dot{\rho}(x)=\sum_{i} \dot{E}_{i}(x)-U_{i}^{\dagger}(x-i) \dot{E}_{i}(x-i) U_{i}(x-i) .
$$

(The contribution from the time derivative of $U$ cancels here because it is generated by, and hence commutes with, $E$, and cancels between $U$ and $U^{\dagger}$.)

First, we know that

$$
\sum_{i} \frac{\partial H_{\mathrm{KS}}}{\partial U_{i}(x)}-U_{i}^{\dagger}(x-i) \frac{\partial H_{\mathrm{KS}}}{\partial U_{i}(x-i)} U_{i}(x-i)=0,
$$

which is why Gauss' law is preserved for the classical lattice system without particles. And the contribution of $j$ to $\dot{E}$ cancels $\dot{\rho}$ in (18) precisely because $\rho$ and $j$ form a conserved current. So Gauss' law is indeed conserved. Alternately, the abrupt change to $\rho(x)$ and $\rho(x+i)$ when a particle passes from being closest to $x$ to being closest to $x+i$ stipulates the change to $E_{i}(x)$ so that Gauss' law will still hold at both sites.

Physically, what this update of $E$ means is that when a particle leaves one box, the charge within that box abruptly drops, and the flux of $E$ field out of the box must abruptly drop too. Meanwhile, the neighboring box, which received the particle, must have the flux of $E$ field out of it abruptly rise. The way to do this is to abruptly change the $E$ field going from the first box to the second, by an amount equal to minus the charge, see Fig. 2. We could also do it by changing the $E$ fields along the other links leading out of these boxes, but this would spoil Gauss' law at the sites at the other ends of those links and is therefore forbidden. Those links will change, in time, via the Hamiltonian evolution of the classical Yang-Mills system.

We should mention that in the abelian theory, one can construct more elaborate Gauss law preserving ways to change $E$, which boil down to moving some of the charge along indirect paths between $x$ and $x+i$. But these are actually not allowed in the nonabelian 
theory, because the charge transforms nontrivially. To satisfy Gauss' law, the particle charge $q$ must also be updated by splitting it up and parallel transporting it along those indirect paths; but when they are added together in the final box, they will not all "point in the same Lie algebra direction", so the magnitude of the sum will have changed. Similar problems arise if one tries to define the charge of a particle as being spread over nearby points, rather than residing entirely at the nearest point. Deriving a local, Gauss' law preserving, $C, P$, and $T$ symmetric alternative to our update proposal is highly nontrivial in the nonabelian context.

Before going further we should make a comment about the normalization of fields which we have adopted. Since $U$ is a parallel transporter it has been most convenient to use the normalization of electric fields in which the Hamiltonian contains a $1 / g^{2} \times E^{2}$, that is our electric field is $g a^{2} E_{\text {cont }} / 2$. The $1 / g^{2}$ in front of the Hamiltonian will be absorbed into the temperature, $\beta_{\mathrm{L}}=4 /\left(g^{2} a T\right)$, which is customary in the 3-D lattice literature [17]. Also the particle charge $Q^{2}$ will convert into "usual" continuum charge as $Q^{2}=g^{4} q^{2} / 4$. It will be convenient to make the particle momentum appear in the Hamiltonian as $\beta_{\mathrm{L}} H=$ $\beta_{\mathrm{L}}\left(H_{\mathrm{KS}}+\sum_{\alpha}|p|_{\alpha}\right)$, so that it can be directly compared with electric field energies. It is then related to physical units by $p_{\text {latt }}=p_{\text {cont }} \times g^{2} a / 4$. Though these normalizations seem strange, they scale out all dimensionful quantities and make the numerical theory have the least awkward inter-relations.

It remains to define the update of $p$. This will be almost uniquely specified by the requirement that the system be Hamiltonian. Naively one would expect the influence of the electric field on $p$ to be

$$
\dot{p}_{i}=q^{a} E_{i}^{a}
$$

with $E_{i}$ chosen to be $E$ on the nearest $i$ type link; but this is wrong, as the system energy then has a time derivative of $p_{i} q E_{i} /|p|$, which cannot be removed by a corresponding change in $E$ because that would spoil Gauss' law. The energy conserving update

$$
\frac{d p_{i}}{d t}=q^{a} E_{i}^{a}-p_{i} \frac{p_{j} q^{a} E_{j}^{a}}{p^{2}}
$$

does not preserve the phase space measure and is also disallowed; in fact, except at the instant when a particle crosses a boundary, measure and energy conservation restrict the allowed changes to $p$ to a rotation about some adjoint charged vector $B$, which must be odd under $C, P$, and $T$;

$$
\dot{p}_{i}=f\left(p^{2}\right) \epsilon_{i j k} p_{j} B_{k}^{a} q^{a} .
$$

So a rotation about the magnetic field is allowed, but not required, to preserve energy and the phase space measure.

However, when a particle crosses the face of the dual lattice separating two points $x$ and $x+i$ and induces a current $j_{i}(x)$, it changes the energy in the electric field $E_{i}(x)$ by

$$
\Delta(\text { energy })=\Delta\left(E^{2} / 2\right)=-E_{i}\left(x, t_{\text {cross }}-0\right) \cdot q+q^{2} / 2=-E_{i}\left(x, t_{\text {cross }}+0\right) \cdot q-q^{2} / 2,
$$

and $p$ must be changed to balance this energy. If only $p_{i}$ changes, from $p_{i \text {,init }}$ to $p_{i \text {,fin }}$, then energy conservation is

$$
\sqrt{p_{i, \mathrm{fin}}^{2}+p_{\perp}^{2}}+\Delta\left(E^{2} / 2\right)=\sqrt{p_{i, \text { init }}^{2}+p_{\perp}^{2}}
$$


which is solved by

$$
p_{i, \text { fin }}=\operatorname{sign}\left(p_{i, \text { init }}\right) \sqrt{\left(|p|_{\text {init }}-\Delta\left(E^{2} / 2\right)\right)^{2}-p_{\perp}^{2}}
$$

if both $|p|_{\text {init }}-\Delta\left(E^{2} / 2\right) \geq 0$ and the argument of the square root is $\geq 0$; otherwise there is no solution, the crossing is energetically forbidden. If the crossing is energetically forbidden, we should set $p_{i \text {,fin }}=-p_{i \text {,init }}$, no crossing occurs, no current is generated, and the particle turns. Otherwise, $p_{i \text {,fin }}$ is taken from (25) above, the current flows, and the particle crosses the boundary. The case where the crossing is from $x+i$ to $x$ follows from this case and parity symmetry.

This proposed update for $p$ conserves energy. What about symplectic measure? The measure is clearly preserved at all times that no particle crosses a boundary, since the evolution of $E, U$ is Hamiltonian, $\dot{\xi}_{\alpha}$ depends on $p_{\alpha}$ but not $\xi_{\alpha}$, and $q$ and $p$ do not change (except for allowed rotations of $p$ ). When a particle reflects, the change $p_{i} \rightarrow-p_{i}$ also preserves the measure. The only nontrivial case is when a particle crosses a boundary. The rotation of $q_{\alpha}$ preserves the measure on $\operatorname{LSU}(2)$ and the change $E \rightarrow E-q$ preserves the measure for $E$ since the change is independent of $E$, which is defined on a vector space. We need only check if the particle phase space measure $\prod_{i} d p_{i} d \xi_{i}$ is preserved, that is, whether

$$
\operatorname{Det}\left[\begin{array}{ll}
\partial p_{i, \text { fin }} / \partial p_{j, \text { init }} & \partial p_{i, \text { in }} / \partial \xi_{j, \text { init }} \\
\partial \xi_{i, \text { fin }} / \partial p_{j, \text { init }} & \partial \xi_{i, \text { fin }} / \partial \xi_{j, \text { init }}
\end{array}\right]
$$

equals 1 . Since the change we are discussing is instantaneous, $\partial \xi_{i \text {,fin }} / \partial p_{j \text {,init }}=0$ and the determinant reduces to blocks,

$$
\operatorname{Det}\left[\begin{array}{ll}
\partial p_{i, \text { in }} / \partial p_{j, \text { init }} & \partial p_{i, \text { fin }} / \partial \xi_{j, \text { init }} \\
\partial \xi_{i, \text { fin }} / \partial p_{j, \text { init }} & \partial \xi_{i, \text { fin }} / \partial \xi_{j, \text { init }}
\end{array}\right]=\operatorname{Det}\left[\frac{\partial \xi_{i, \text { in }}}{\partial \xi_{j, \text { init }}}\right] \operatorname{Det}\left[\frac{\partial p_{i, \text { fin }}}{\partial p_{j, \text { init }}}\right]
$$

and we need to show that these determinants are inverses.

Without loss of generality we consider the case in which $p_{1}$ changes, with $p_{1}>0$. We

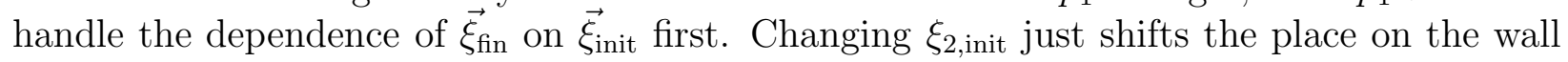
where the particle crosses, but $\vec{p}$ is the same at all times; so only $\xi_{2 \text {,fin }}$ is changed, by the same amount as the initial change. The same holds for $\xi_{3}$. However, changing $\xi_{1 \text {,init }}$ by $d \xi_{1}$ changes the arrival time at the wall by $-d \xi_{1} / v_{1 \text {,init }}$. The particle propagates at $\vec{v}_{\text {fin }}$ rather than $\vec{v}_{\text {init }}$ for $d \xi_{1} / v_{1, \text { init }}$ longer than without the change, leading to a change in the final positions equal to $\left(\vec{v}_{f i n}-\vec{v}_{\text {init }}\right) d \xi_{1} / v_{1, \text { init }}$, plus the change of $d \xi_{1}$ in $\xi_{1, \text { fin }}$. Hence,

$$
\operatorname{Det}\left[\frac{\partial \xi_{i, \text { fin }}}{\partial \xi_{j, \text { init }}}\right]=\operatorname{Det}\left[\begin{array}{ccc}
v_{1, \text { fin }} / v_{1, \text { init }} & 0 & 0 \\
\left(v_{2, \text { fin }}-v_{2, \text { init }}\right) / v_{1, \text { init }} & 1 & 0 \\
\left(v_{3, \text { fin }}-v_{3, \text { init }}\right) / v_{1, \text { init }} & 0 & 1
\end{array}\right]=\frac{v_{1, \text { fin }}}{v_{1, \text { init }}} .
$$

Now for the dependence of $\vec{p}_{\text {fin }}$ on $\vec{p}_{\text {init }}$. Since $p_{2, \text { init }}=p_{2 \text {,fin }}$ and similarly for $p_{3}$, only the change in the final value of $p_{1, \text { fin }}$ need be computed. Taking the appropriate derivatives of (25) gives

$$
\begin{aligned}
\operatorname{Det}\left[\frac{\partial p_{i, \text { fin }}}{\partial p_{j, \text { init }}}\right] & =\operatorname{Det}\left[\begin{array}{ccc}
\frac{p_{1, \text { init }}}{p_{1, \text { fin }}}\left(\frac{|p|_{\text {init }}-\Delta\left(E^{2} / 2\right)}{|p|_{\text {init }}}\right) & -\frac{\Delta\left(E^{2} / 2\right) p_{2}}{p_{1, \text { fin }}|p|_{\text {init }}} & -\frac{\Delta\left(E^{2} / 2\right) p_{3}}{p_{1, \text { fin }}|p|_{\text {init }}} \\
0 & 1 & 0 \\
0 & 0 & 1
\end{array}\right] \\
& =\frac{p_{1, \text { init }}}{p_{1, \text { fin }}}\left(\frac{|p|_{\text {init }}-\Delta\left(E^{2} / 2\right)}{|p|_{\text {init }}}\right) .
\end{aligned}
$$


Now observe that $|p|_{\text {init }}-\Delta\left(E^{2} / 2\right)=|p|_{\text {fin }}$ and that $p_{1} /|p|=v_{1}$; so the two determinants are inverses and the measure is indeed conserved. The cases where $p_{2}$ or $p_{3}$ change, or where the particle moves in the opposite direction, follow from cubic and parity symmetry.

If we had allowed $p$ in the orthogonal directions to change, it generically would not preserve the measure, so the choice of update is almost unique.'t

The first four conditions for a valid update rule have specified the update uniquely except for the freedom to perform rotations of the form shown in (22). It would seem natural to define the magnetic field $B_{i}^{a}$ at a point in terms of the 3 nearest plaquettes p $^{\text {and to rotate }}$ momenta according to

$$
\dot{p}_{i}=\epsilon_{i j k} \frac{p_{j}}{|p|} B_{k}^{a} q^{a} .
$$

As we discussed, the magnetic term in Wong's equations plays no role in reproducing the right hard thermal loops for a plasma close to equilibrium. Thus we are free to omit this rotation when dealing with quasi-equilibrium processes.

Next let us verify that the continuum limit of our update rules give Wong's equations. This is known for the classical field equations without particles evolving under the KogutSusskind Hamiltonian, so we need only check the terms involving particles. The update of the particle charge is explicitly the adjoint parallel transport along its trajectory, as it should be. The current in the $i$ direction from a particle occurs in jolts of magnitude $q$ and frequency $1 / v_{i}$, so the time averaged current is $q v_{i}$ as it should be. The impulse on a moving particle is of magnitude $\Delta p_{i}=p_{i, \mathrm{fin}}-p_{i \text {,init }}$; expanding (23) and (25) to leading order in $q$ gives $\Delta p_{i}=q \cdot E_{i} / v_{i}$, and such impulses also occur with frequency $1 / v_{i}$, so the time averaged force on the particle is $q \cdot E_{i}$, also as it should be. We will explore the corrections to the plasma frequency, due to the discrete nature of the current and the "kicks," in Appendix A. Wong's equations are recovered for the motion of a particle through slowly varying fields, if $q$ is sufficiently small.

We still must check whether the thermodynamics of this system are correct; we will do this in the next chapter. But first, let us review and summarize the update rule. The fields evolve under the Kogut-Susskind Hamiltonian and the particles move freely, except at such exact instants when a particle crosses a boundary (a face of the dual lattice), i.e. when it goes from being nearest one point to nearest another. The charge is then parallel transported by the link operator dual to the face, and the electric field on that link is abruptly changed by $-q$ (or $+q$ if the motion is in the - direction). The particle momentum parallel to the link is changed to cancel the energy change of the electric field. If that is energetically impossible, the particle momentum parallel to the link is flipped and no crossing, or change to $q, E$, occurs. This update is Hamiltonian with total energy

$$
\text { Energy }=\sum_{x, i} \frac{E_{i}^{2}(x)}{2}+\sum_{\square}\left(1-\frac{1}{2} \operatorname{Tr} U_{\square}\right)+\sum_{\alpha}|p|_{\alpha},
$$

and preserves Gauss' law identically. It is also manifestly gauge, $C, P, T$, and cubic invariant.

\footnotetext{
${ }^{4} \mathrm{~A}$ rotation of $p_{\perp}$ violates parity unless its magnitude depends on $B$ or some other $P$ odd field; and an energy conserving change in the magnitudes of $p_{1}$ and $p_{\perp}$ will have nonunity Jacobian.

${ }^{5}$ One should be careful here to make the choice of plaquettes in a way which is preserved under parity and the cubic point group.
} 


\section{Thermodynamics of the lattice system}

In the last section we have proposed a continuum time system and its update rule and have shown that it is Hamiltonian, ie that the update rule preserves energy and phase space measure. It therefore has well defined thermodynamics, which we now explore. The canonical partition function is

$$
\begin{aligned}
Z & =\int \prod_{\alpha} d^{3} \xi_{\alpha} d^{3} p_{\alpha} d q_{\alpha} \prod_{i, x} d E_{i}(x) d U_{i}(x) \prod_{x} \delta\left(-\rho(x)+D_{\mathrm{L}} \cdot E(x)\right) e^{-\beta_{\mathrm{L}} H}, \\
H & =\sum_{x, i} \frac{E_{i}^{2}(x)}{2}+\sum_{\square}\left(1-\frac{1}{2} \operatorname{Tr} U_{\square}\right)+\sum_{\alpha}|p|_{\alpha} .
\end{aligned}
$$

The appropriate measures, ie the Haar measure for $U$ and the Lebesgue measure on LSU(2) restricted to $q^{2}=Q^{2}$ for $q$, are implied. The $\delta$ function enforces Gauss' law at each point and the meanings of $\rho$ and $D_{\mathrm{L}} \cdot E$ are as previously defined. The quantity $\beta_{\mathrm{L}}$ here combines all the dimensionful parameters of the lattice system, $\beta_{\mathrm{L}}=4 / g^{2} a T$; when it is large we are on a fine lattice, or at weak coupling, which is equivalent in the classical theory.

Note first that the kinetic momenta will obey a Boltzmann distribution and are independent of all other degrees of freedom; the partition function factorizes and if we are interested in the thermodynamics of the IR classical gauge fields then we may integrate out $p$. Note also that $\xi$ and $q$ do not appear in the Hamiltonian; their only influence is in determining $\rho$ appearing in Gauss' law.

It is most convenient to enforce Gauss' law with an adjoint valued Lagrange multiplier $A_{0}$ [17],

$$
\prod_{x}\left(D_{\mathrm{L}} \cdot E(x)-\rho(x)\right)=\int \prod_{x} d A_{0}(x) \exp \left[i \beta_{\mathrm{L}} \sum_{x} A_{0}^{a}(x)\left(D_{\mathrm{L}} \cdot E^{a}(x)-\rho^{a}(x)\right)\right],
$$

which makes the electric fields quadratic; the $E$ integral may now be performed, generating a kinetic term for $A_{0}$. The partition function reduces to

$$
\begin{aligned}
Z & =\prod_{i, x} d U_{i}(x) d A_{0}(x) e^{-\beta_{\mathrm{L}} H_{U A}} I\left(A_{0}\right), \\
H_{U A} & =\sum_{\square}\left(1-\frac{1}{2} \operatorname{Tr} U_{\square}\right)+\frac{1}{2} \sum_{x}\left(D_{\mathrm{L}} A_{0}\right)^{2}(x), \\
I\left(A_{0}\right) & =\int \prod_{\alpha} d^{3} \xi_{\alpha} d q_{\alpha} e^{-i \beta_{\mathrm{L}} \sum_{x} A_{0}(x) \rho(x)},
\end{aligned}
$$

where the meaning of $\left(D_{\mathrm{L}} A_{0}\right)^{2}$ should be clear. The particles enter in the last term, which only depends on $A_{0}$.

Now let us compute $I\left(A_{0}\right)$. Since $\rho$ is the sum of $\rho_{\alpha}$ from each particle, $\exp -i \beta_{\mathrm{L}} A_{0} \rho$ is the product over $\alpha$ of $\exp -i \beta_{\mathrm{L}} A_{0} \rho_{\alpha}$; the integral factorizes into an integral over each particle,

$$
I\left(A_{0}\right)=\prod_{\alpha}\left[\int d q_{\alpha} \int d^{3} \xi_{\alpha} \exp \left(-i \beta_{\mathrm{L}} \sum_{x} A_{0}^{a}(x) q_{\alpha}^{a} \times\left\{\begin{array}{ll}
1 & \xi \text { at } x \\
0 & \xi \text { not at } x
\end{array}\right)\right] .\right.
$$


The integral over $\xi$, normalized so $\int d \xi=1$, gives a sum over sites of a term where the particle is at that site,

$$
I\left(A_{0}\right)=\prod_{\alpha}\left[\frac{1}{N^{3}} \sum_{x} \int d q_{\alpha} \exp \left(-i \beta_{\mathrm{L}} A_{0}^{a}(x) q_{\alpha}^{a}\right)\right] .
$$

Now using

$$
\int d q \exp \left(-i \beta_{\mathrm{L}} A_{0}^{a}(x) q^{a}\right)=\frac{\sin \left(\beta_{\mathrm{L}} Q \sqrt{A_{0}^{2}(x)}\right)}{\beta_{\mathrm{L}} Q \sqrt{A_{0}^{2}(x)}},
$$

(normalizing so that $\int d q=1$ ) and performing the product, we arrive at

$$
I\left(A_{0}\right)=\left[\frac{1}{N^{3}} \sum_{x} \frac{\sin \left(\beta_{\mathrm{L}} Q \sqrt{A_{0}^{2}(x)}\right)}{\beta_{\mathrm{L}} Q \sqrt{A_{0}^{2}(x)}}\right]^{N_{\mathrm{p}}} .
$$

The above expression is exact but not very insightful as written. It is best to take a thermodynamic limit and to expand the expression in the sum. Denote by $\langle$ ARG $\rangle$ the mean value of the argument of the sum in (41). Rescale $I\left(A_{0}\right)$ by $\langle\mathrm{ARG}\rangle^{-N_{\mathrm{p}}}$, which just changes the normalization of the partition function, and write it as

$$
I\left(A_{0}\right)=\left[1+\left(-1+\frac{1}{N^{3}\langle\mathrm{ARG}\rangle} \sum_{x} \frac{\sin \left(\beta_{\mathrm{L}} Q \sqrt{A_{0}^{2}(x)}\right)}{\beta_{\mathrm{L}} Q \sqrt{A_{0}^{2}(x)}}\right)\right]^{N_{\mathrm{p}}} .
$$

As $N^{3} \rightarrow \infty$ and $N_{\mathrm{p}} \rightarrow \infty$ with $N_{\mathrm{p}} / N^{3} \equiv\langle n\rangle$ fixed, the term in round parenthesis vanishes as $N^{-3 / 2}$, and we may use the approximation

$$
\left(1+\frac{x}{N}\right)^{N}=\exp (x) \exp \left(-x^{2} / 2 N\right) \times\left(1+O\left(x^{3} / N^{2}\right)\right) .
$$

The $\exp \left(-x^{2} / 2 N\right)$ term in the identity means that there is a very weak nonlocal interaction term between fluctuations from the mean value of $A_{0}^{2}$ at pairs of points, trying to force the global average of $A_{0}^{2}$ towards its equilibrium value. It is probably safe to ignore this term, and higher corrections strictly vanish in the large $N$ limit. Neglecting the nonlocal term, we get

$$
I\left(A_{0}\right) \simeq \exp \left(\frac{\langle n\rangle}{\langle\mathrm{ARG}\rangle} \sum_{x} \frac{\sin \left(\beta_{\mathrm{L}} Q \sqrt{A_{0}^{2}(x)}\right)}{\beta_{\mathrm{L}} Q \sqrt{A_{0}^{2}(x)}}\right) .
$$

Since we are typically interested in a system where $N^{3} \sim 10^{4}$ and $N_{\mathrm{p}}>10^{5}$, the thermodynamic limit is justified.

Now, expanding the term in the sum,

$$
\frac{\sin \left(\beta_{\mathrm{L}} Q \sqrt{A_{0}^{2}(x)}\right)}{\beta_{\mathrm{L}} Q \sqrt{A_{0}^{2}(x)}}=1-\frac{\beta_{\mathrm{L}}^{2} Q^{2} A_{0}^{2}(x)}{6}+\frac{\beta_{\mathrm{L}}^{4} Q^{4}\left(A_{0}^{2}\right)^{2}(x)}{120}-\ldots
$$


we see that $I\left(A_{0}\right)$ contains a Debye screening term and quartic and higher self-interaction terms for $A_{0} ; I\left(A_{0}\right)$ becomes $\exp \left(-\beta_{\mathrm{L}} V\left(A_{0}^{2}\right)\right)$, where the potential $V$ is

$$
\begin{aligned}
V\left(A_{0}^{2}\right) & =\frac{m_{\mathrm{D}}^{2}}{2} \sum_{x} A_{0}^{2}+\frac{\lambda_{\mathrm{A}}}{4} \sum_{x}\left(A_{0}^{2}\right)^{2}+\ldots, \\
m_{\mathrm{D}}^{2} & =\frac{\langle n\rangle \beta_{\mathrm{L}} Q^{2}}{3\langle\mathrm{ARG}\rangle} \\
\lambda_{\mathrm{A}} & =-\frac{\langle n\rangle \beta_{\mathrm{L}}^{3} Q^{4}}{30\langle\mathrm{ARG}\rangle}, \ldots
\end{aligned}
$$

Finally,

$$
\langle\mathrm{ARG}\rangle=1-\frac{\beta_{\mathrm{L}}^{2} Q^{2}}{6}\left\langle A_{0}^{2}\right\rangle+O\left(\beta_{\mathrm{L}}^{4} Q^{4} A_{0}^{4}\right)
$$

$\left\langle A_{0}^{2}\right\rangle$ is UV dominated and well approximated by its perturbative value. At lowest order, $\left\langle A_{0}^{2}\right\rangle=3 \Sigma / 4 \pi \beta_{\mathrm{L}}$, with $\Sigma \simeq 3.1759$ [10]. In practice it is necessary to make $Q \sim \beta_{\mathrm{L}}^{-1}$, see below, in which case, for $\beta_{\mathrm{L}} \sim 10,\langle\mathrm{ARG}\rangle$ typically differs from 1 by $\sim 1 \%$. As we mentioned in Section 2 , the ratio $m_{\mathrm{D}}^{2} /\langle n\rangle$ should equal $\left\langle 2 q_{1}^{2}\right\rangle\left\langle E^{-1}\right\rangle=\left(Q^{2} / 3\right)\left(2 \beta_{L} / 2\right)$ for classical particles in the continuum. Discretization has shifted this by a small $O\left(Q^{2}\right)$ correction.

In a group other than $\mathrm{SU}(2)$, the calculation begins to go differently at Eq. (40), and the final Debye mass is different. For instance, in $\mathrm{U}(1)$ gauge theory, the integral there gives $\cos \left(\beta_{\mathrm{L}} Q A_{0}\right)$, and $m_{\mathrm{D}}^{2}$ and $\lambda_{\mathrm{A}}$ are 3 and 5 times larger, respectively.

We have now shown that the thermodynamics are the same as lattice regulated YangMills theory in the dimensional reduction approximation, at a specific value of Debye mass, except for the added higher order interaction terms for the $A_{0}$ field. A minimal requirement for the thermodynamics to be all right is that the $A_{0}$ field is not strongly coupled, $\lambda_{\mathrm{A}} \leq 1$. The lattice $\lambda_{\mathrm{A}}$ corresponds to the physical $4 \lambda_{\mathrm{A}} / g^{2}$, so this requirement is that the coupling of the $A_{0}$ sector is weaker than the coupling of the gauge sector, which should be sufficient since the $A_{0}$ field is quite massive. This requirement is roughly

$$
Q^{4}\langle n\rangle<\frac{30}{\beta_{\mathrm{L}}^{3}} .
$$

In fact it will turn out that dynamic considerations require that $Q$ be on order or smaller than $\beta_{\mathrm{L}}^{-1}$, in which case, unless $\langle n\rangle$ is very large, $\lambda_{\mathrm{A}}$ will be very small, as it should be in the dimensionally reduced Hamiltonian.

We should also require that the Debye screening mass from particles be larger than that from hard lattice modes (as otherwise the hard thermal loops are dominated by wrong lattice mode contributions, rather than right particle contributions), in which case

$$
\frac{12 \Sigma}{\pi \beta_{\mathrm{L}}^{2}}<\langle n\rangle Q^{2}
$$

However, it is not necessary on thermodynamic grounds to make $m_{\mathrm{D}}^{2}$ small in lattice units. When $m_{\mathrm{D}}^{2}$ is large in physical units, ie $m_{\mathrm{D}}^{2} \gg \beta_{\mathrm{L}}^{-2}$ in lattice units, then the influence of the $A_{0}$ field on the vector fields is perturbative. Making $m_{\mathrm{D}}^{2}$ on order the lattice spacing complicates the problem of integrating out the $A_{0}$ field to find its influence on the infrared physics, but the 
one loop integration can still be performed; we treat this problem in Appendix B. However, it will turn out on dynamical grounds that one should not make the Debye mass too large, as the plasma frequency then takes on lattice artifact corrections, discussed in Appendix A.

\section{Discrete time update algorithm}

In this section we give a detailed description of a stable and accurate discrete time update algorithm to solve the equations of motion for the particles and lattice fields. Probably most readers can safely skip this section; nothing in it is essential for understanding the rest of the paper, although it certainly is important that a stable and well behaved algorithm exists for implementing the system described in the last two sections.

The numerical algorithm we will construct is essentially a leapfrog, with modifications to meet the needs of the specific problem under investigation. In particular we make sure the algorithm is time centered, and the particle update part is exactly energy conserving. The stepsize errors in the algorithm should be $O\left((\Delta t)^{2}\right)$ where $\Delta t$ is the time step in lattice units.

The variables to be updated are: $\left(\xi_{\alpha}, p_{\alpha}, q_{\alpha}\right)$ and $\left(E_{x, i}, U_{x, i}\right)$. If the connections $U$ were fixed and the particles did not rotate due to magnetic fields, then we could perform the update (of $E, p, q, \xi$ ) exactly, as follows. Starting with $E, p, \xi, q$ at time $t$, we project where $\xi$ will be at time $t+\Delta t$,

$$
\xi_{\text {proj }, \alpha, i}(t+\Delta t)=\xi_{\alpha, i}(t)+\Delta t \frac{p_{\alpha, i}}{|p|_{\alpha}}
$$

For all $\alpha$ where the projected location is in the same box (dual lattice cell) as the initial location, ie no boundary is crossed, then $\xi(t+\Delta t)$ equals the projection, and we update $\xi$. For other points, we draw the straight line path between $\xi(t)$ and $\xi_{\text {proj }}$ and find the first boundary it crosses. $\xi$ can be updated to this point, $\xi_{\text {bound }}$, which it reaches at time $t_{\text {bound }}=t+\left|\xi_{\text {bound }}-\xi(t)\right|$. At this point we must be careful, because more than one particle may interact with the same electric field, and the updates will depend on the order of interaction. So we order all particles which cross a boundary according to crossing time; then we solve the crossing conditions in that order, modifying $E, q$, and $p$ as discussed in Section 3. For each particle, after these variables are updated, the particle's position at time $t+\Delta t$ is again projected, starting at $\xi_{\text {bound }}$ and using the new $p$. If it crosses another boundary before time $t+\Delta t$, we again update it to the boundary, compute the time of arrival, and insert it at the place appropriate for the new crossing time in the collection of particles to be updated; otherwise we update it to its projected position at time $t+\Delta t$. When the last crossing has been dealt with, then all $E, p, q$, and $\xi$ have been updated to time $t+\Delta t$. This algorithm is exact except for roundoff errors.

One comment is in order about this update. Ordering the particles by time of crossing takes $O\left(N_{\mathrm{p}} \ln N_{\mathrm{p}}\right)$ steps, so the algorithm does not quite scale linearly with volume. In practice, though, the ordering takes $C \Delta t N_{\mathrm{p}} \ln N_{\mathrm{p}}$ computations with $C$ a fairly small number, and this part of the algorithm takes less time than formally $O\left(N_{\mathrm{p}}\right)$ or $O\left(N^{3}\right)$ parts.

Now we must incorporate the above idea into the leapfrog update of the $E, U$ fields. The 
leapfrog in the absence of particles is

$$
\begin{aligned}
U_{i}(x, t+\Delta t / 2) & =\exp \left(i \Delta t \tau^{a} E_{i}^{a}(x, t)\right) U_{i}(x, t-\Delta t / 2), \\
E_{i}^{a}(x, t+\Delta t) & =E_{i}^{a}(x, t)-\Delta t \frac{\partial H_{\mathrm{KS}}(U(t+\Delta t / 2))}{\partial U_{i}(x, t+\Delta t / 2)},
\end{aligned}
$$

where the meaning of $\partial H_{\mathrm{KS}} / \partial U$ is defined between (16) and (18). If one needed to define $E_{i}^{a}(x, t+\Delta t / 2)$ one could do so by applying only half of the update, (54).

We combine this leapfrog and the update of $\xi$ etc. discussed above, as follows.

1. Start with $E(t), p(t), q(t), \xi(t)$, and $U(t-\Delta t / 2)$.

2. Determine $U(t+\Delta t / 2)$ according to (53).

3. Apply half the $E, U$ leapfrog update of $E$; namely, set

$$
E_{i}^{a}(x, t+0)=E_{i}^{a}(x, t)-\frac{\Delta t}{2} \frac{\partial H_{\mathrm{KS}}(U(t+\Delta t / 2))}{\partial U_{i}(x, t+\Delta t / 2)},
$$

Also update $p(t)$ to $p(t+0)$ by rotating about $B$ for time $\Delta t / 2$; first set

$$
p_{\text {temp }, \alpha, i}=p_{\alpha, i}+\frac{\Delta t}{2} \epsilon_{i j k} \frac{p_{\alpha, j}}{|p|_{\alpha}} B_{k}^{a}(\xi) q^{a} .
$$

(Here $B_{k}(\xi)$ means the $i, j$ plaquette which is closest to the point $\xi$ and with indicies living at the point where the indicies of $q$ reside.) Then rescale $p$ to its original magnitude,

$$
p_{\alpha, i}(t+0)=p_{\text {temp }, \alpha, i} \frac{|p|_{\alpha}(t)}{|p|_{\text {temp }, \alpha}} .
$$

4. Update $E, q, \xi$, and $p$ from time $t+0$ to time $t+\Delta t-0$ using the "fixed connection, no $B$ field" algorithm presented above, and $U=U(t+\Delta t / 2)$.

5. Apply the other half of the $E, U$ leapfrog,

$$
E_{i}^{a}(x, t+\Delta t)=E_{i}^{a}(x, t+\Delta t-0)-\frac{\Delta t}{2} \frac{\partial H_{\mathrm{KS}}(U(t+\Delta t / 2))}{\partial U_{i}(x, t+\Delta t / 2)},
$$

and rotate the momenta as in step 3. Now, return to 1., but with the value of $t$ incremented by $\Delta t$.

Applying these in order is one leapfrog update. Note that the update is time symmetric and exactly Gauss constraint preserving at each step, and that step 4. exactly conserves energy. The overall conservation of energy is exactly as good as in the Kogut-Susskind leapfrog algorithm, that is, energy fluctuates by a small $O\left((\Delta t)^{2} N^{3 / 2}\right.$ ) amount (which for a $20^{3}$ grid at $\Delta t=0.05$ and 30 particles per site is less than a part in $10^{5}$ of the total energy) and the central value of the energy is absolutely stable.

As we have discussed, the effect of magnetic fields on particles does not contribute to the hard thermal loops for a plasma close to equilibrium. So that part of steps 3. and 5. can be 
left out when studying quasi-equilibrium processes, which in practice saves at least $1 / 3$ of the update time.

Finally we present a canonical ensemble thermalization algorithm for this system. It is a straightforward generalization of the "constrained molecular dynamics" algorithm of [42]. Beginning from an arbitrary choice of $U(\Delta t / 2), \xi(0)$, and $q(0)$, we choose $p(0+0)$ from the Boltzmann distribution at some inverse temperature $\beta_{\mathrm{L}}$ and $E(0+0)$ from the Gaussian distribution at the same temperature, modulo the Gauss constraints. As in 42 this is done by choosing $E$ without regard to the constraints and then orthogonally projecting to the constraint surface, which correctly thermalizes the transverse components of $E$ and correctly enforces the constraints on the longitudinal components. The algorithm is identical to that in 42] except that the particle contribution to Gauss' law must be added. This chooses $p, E$ with thermal weight from the fixed $U, \xi, q$ subspace of phase space. Then we evolve the system under the Hamiltonian evolution, using the algorithm presented above, for some length of time, at the end of which we again draw $E$ and $p$ from the thermal ensemble; we repeat until the (athermal) original information in the $U, \xi$, and $q$ has been destroyed and measurables attain values which do not change in the mean under Hamiltonian evolution or further thermalization. The spirit of this algorithm is that of a molecular dynamics MonteCarlo. Note that it is important to choose $E$ and $p$ at some time between $t+0$ and $t+\Delta t-0$ and not at $t$ or $t+\Delta t$ because the half update from $t-0$ to $t$ changes $E$ from being Gaussian and uncorrelated with $\partial H_{\mathrm{KS}} / \partial U$ to being correlated with $\partial H_{\mathrm{KS}} / \partial U$. (Similarly in 42 it was necessary to choose $E$ defined at the same time as $U$ and to perform a half update before beginning the leapfrog.)

We should also note that there is no obstacle to applying a Langevin type thermalization algorithm based on the one developed in [43], and in particular that it is trivial to couple Langevin noise to the particle momenta. This might be important if one wanted to simulate thermalization of these modes through interactions with some other degrees of freedom, for instance strong scattering of fermions in the electroweak model.

\section{Some results for the abelian theory}

Before diving into the study of $N_{\mathrm{CS}}$ diffusion we should check that the particle method is producing the right physics of HTL's. In this section we will first discuss how small $Q^{2}$ must be for the system to give good behavior, and then we will study the abelian theory at suitably small $Q^{2}$ to see if the hard contributions to the retarded self-energy are correct.

We saw in Section 4 that a necessary condition for the theory to have a weakly coupled $A_{0}$ sector is

$$
Q^{4}\langle n\rangle<\frac{30}{\beta_{\mathrm{L}}^{3}} .
$$

In fact, dynamical considerations demand that $Q^{2}$ be still smaller. To get the right hard thermal loop effects in the dynamics, we need a particle to travel a distance longer than the magnetic length scale $1 / g^{2} T$ (or $\beta_{\mathrm{L}}$ in lattice units) before its momentum is randomized by interactions with the plasma. Normally we would expect the dominant randomizing process to be Coulomb scattering from other particles as depicted on the left-hand side of Figure 3; but in fact the dominant processes are absorption or emission of hard classical field 


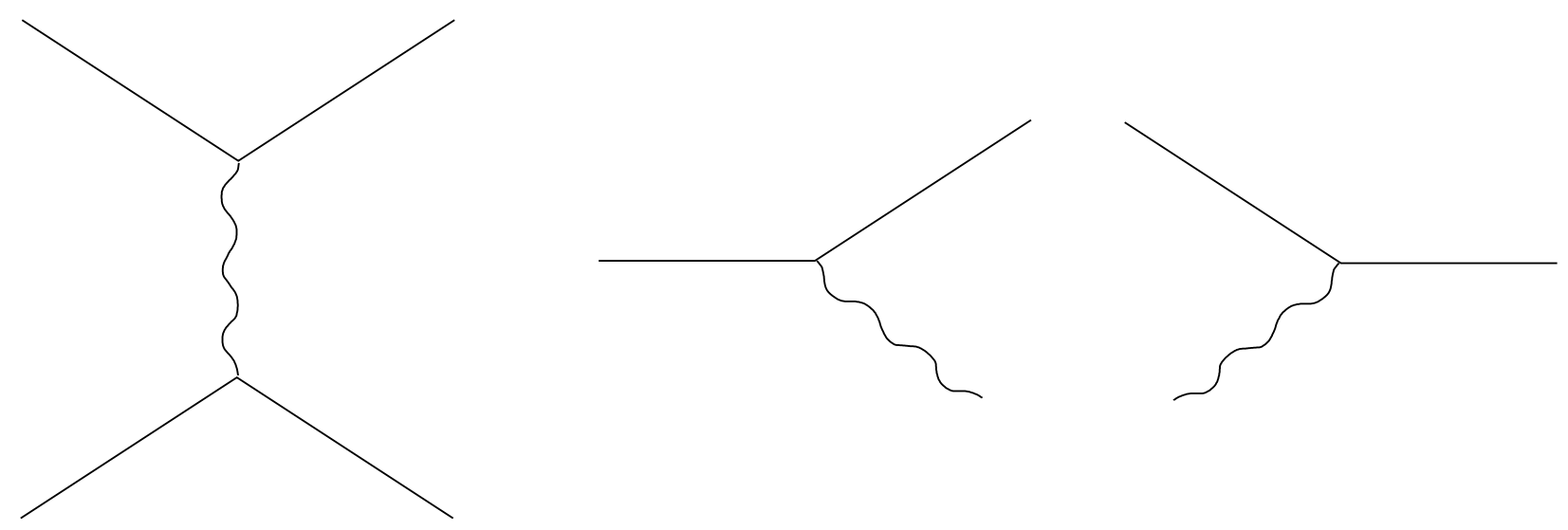

Figure 3: Diagram which naively dominates particle scattering, left, and Cerenkov diagrams which are really dominant, right.

excitations. These processes are not kinematically forbidden because the lattice dispersion relations "turn over" at high momentum, so hard excitations move slower than the speed of light, allowing particles to Cerenkov radiate or absorb. From the point of view of the lattice modes, this is saying that the ultraviolet lattice modes are Landau damped due to the particles, which is possible because their dispersion relation has $\omega / k<1$.

A "worst case" estimate is that the typical electric field which a particle interacts with is uncorrelated with the charge $q$ of the particle, so the momentum $p$ falls by $q^{2} / 2=Q^{2} / 2$ in addition to receiving a kick uncorrelated with its initial value. The average particle (averaging over directions of motion) crosses walls with frequency $3 / 2$ in lattice units; so the decorrelation rate for $p$ under the above approximation is

$$
\text { rate } \sim \frac{3 Q^{2}}{4\langle p\rangle}=\frac{Q^{2} \beta_{\mathrm{L}}}{4}
$$

which must be $\ll \beta_{\mathrm{L}}^{-1}$; so $Q^{2} \ll 4 / \beta_{\mathrm{L}}^{2}$. In practice the above estimate for randomization is close to the real behaivor; for $\beta_{\mathrm{L}}=10$ and $Q=0.08$, the decoherence time is about 80 in lattice units. In our work we typically demand $Q^{2} \leq \beta_{\mathrm{L}}^{-2}$, which forces the number of particles to be quite large, $\langle n\rangle \sim 30$, in order to satisfy (51). This makes the update of particles the dominant numerical cost, but it pushes us closer to the Vlasov equation limit.

Note also that the processes mentioned above make the evolution of the UV classical lattice modes damped and noisy, with a damping strength proportional to $Q^{2}\langle n\rangle$. The consequences for the infrared magnetic sector deserve investigation.

The above behavior is disturbing enough to encourage us to check that the behavior of the infrared degrees of freedom is correct. This is most easily done for the abelian theory, because there the electric field and the current are gauge invariant and one can easily probe the system with external currents and study the response. It would also be possible to do this in the nonabelian theory in a specific gauge, but complications from pure classical gauge theory interactions complicate interpreting results; the abelian theory provides a nice, clean environment to study the dynamics of the particle technique.

We will perform two numerical tests on the abelian theory. First, we verify that the lattice field has the correct dispersion in the infrared. Second, we probe the retarded propagator 


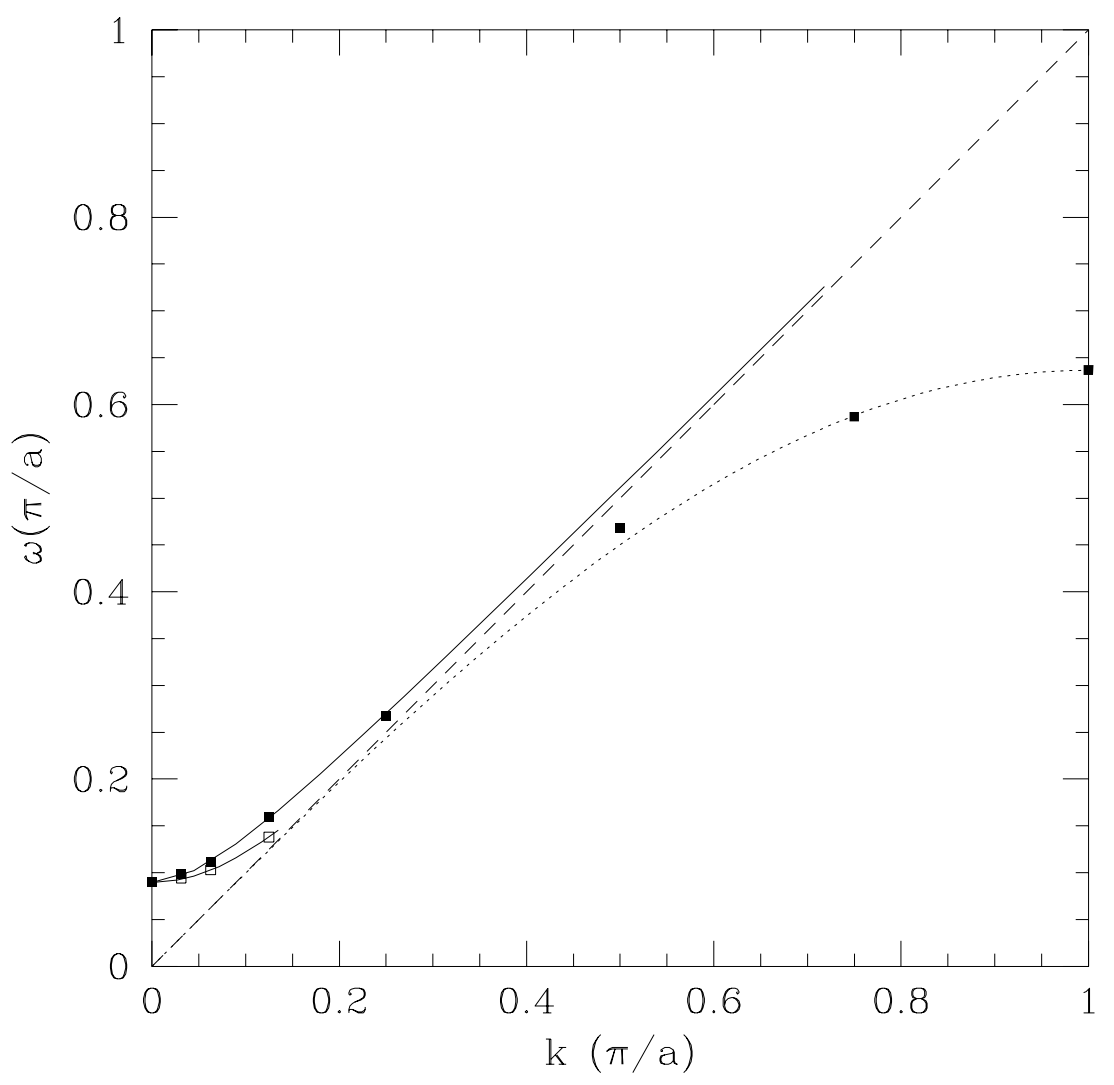

Figure 4: Dispersion relation: free field in the continuum (dashed line: $\omega=k$ ); free field on the lattice (dotted curve: $\omega a=2 \sin (k a / 2)$ ); plasma modes: transverse (theory: the upper solid curve; data: solid rectangles) and longitudinal (theory: the lower solid curve; data: open rectangles).

by studying the linear response of the abelian plasma to an external current.

To measure the plasma dispersion relation, we pick initial conditions so the (spatial) Fourier mode of wave vector $k$ has a large electric field. This sets up a plasma oscillation with this wave number. We evolve the fields and measure the electric field in the same Fourier mode at regular time intervals. Then we Fourier transform this time series and pick out the frequency with the most power; this is the plasma frequency. We have used this procedure to measure the dispersion for both transverse and longitudinal plasma modes. The results are summarized in Figure 1 , where we compare them to the lattice, free field dispersion relation and the continuum dispersion relations, with and without hard thermal loops. These data are for $Q=0.0159, \beta_{\mathrm{L}}=18.86$, and $\langle n\rangle=50$, corresponding to a plasma frequency of $\omega_{\mathrm{p}}=0.282$. (Recall that in the abelian theory $m_{\mathrm{D}}^{2}$ and $\omega_{\mathrm{p}}^{2}$ are 3 times larger in terms of $Q^{2}\langle n\rangle$ than in $\mathrm{SU}(2)$.) The numercal results agree with theory remarkably well in the infrared. As $k$ gets bigger, the data points deviate from the continuum theory curve and bend down to match the lattice dispersion relation. This is expected because hard plasma modes are much less influenced by hard thermal loops and behave like free lattice modes. 
We have also computed the plasma frequency at $k=0$ analytically, in Appendix A. There we conclude that the correction due to lattice artifacts should be negligible for $\omega_{\mathrm{p}}^{2} \ll 1$ in lattice units. This is supported by our numerical results.

To probe the retarded (transverse) photon propagator, we drive the system with an external current of the following form:

$$
j_{i}=\hat{j}_{i} j_{0} \sin (\omega t) \sin (k x),
$$

where the amplitude $j_{0}$ is small so that linear response theory applies. We study response of the plasma and measure the space-time average of $j \cdot E$, which, according to Appendix $\mathbb{C}$, can be written in terms of the transverse photon polarization function:

$$
\langle j \cdot E\rangle=\frac{j_{0}^{2}}{4} \cdot \frac{\omega \Pi_{\mathrm{i}}(\omega, k)}{\left[\omega^{2}-k^{2}-\Pi_{\mathrm{r}}(\omega, k)\right]^{2}+\Pi_{\mathrm{i}}^{2}(\omega, k)},
$$

where $\Pi_{\mathrm{r}}(\omega, k), \Pi_{\mathrm{i}}(\omega, k)$ are the real and imaginary parts of the transverse polarization function, respectively. We also measure the out of phase response of $E$ and the case of longitudinal excitation; the specific expressions are in Appendix C.

We want both to compare to theory and to test the rotational invariance of the particle induced hard thermal loops. To do so, we choose two $k$ vectors which are of the same length but are inequivalent under the cubic point group, and we study the complex propagator for each, at frequencies above and below the plasma resonance. We choose the vectors $k=(3,0,0) \times \pi / 16$ and $k=(2,2,1) \times \pi / 16$ on a $32^{3}$ lattice, with $\beta_{\mathrm{L}}=20, Q^{2}=0.0005$, and $\langle n\rangle=60$ (so $\omega_{\mathrm{p}}=0.447$ ). We excite the plasma at each wave vector and numerous frequencies in turn and integrate the resulting response for long enough to get reasonably clean results.

We present the results, plotted against the theory, in Figure 5. A few comments are in order. In the strict hard thermal loop approximation there would be no imaginary part to the self-energy above the light cone, but the appearance of a small imaginary part, which will arise at $O\left(Q^{4} \beta_{\mathrm{L}}^{2}\langle n\rangle\right)$ since we are in the abelian theory, qualitatively changes the response near the resonance by giving the resonance some width. For the transverse data, we have added a small phenomenological imaginary part to the self-energy chosen to make the theory give a resonance of about the same sharpness as the data. The match between data and theory is very good except that the location of the resonance is shifted a little towards lower $\omega$ in the data. This is expected; the particle response weakens with frequency because the particles cannot respond to a field faster than they encounter it, which is how often they cross a boundary, as we examine quantitatively in Appendix A. Also note that the data for $k \propto(2,2,1)$ (triangles) have their resonance very slightly after the data for $k \propto(3,0,0)$, which is expected from the $O\left(k^{4}\right)$ lattice corrections to the dispersion relations. However, besides this, they are indistinguishable, which is a good check that the particle contribution to the self-energy is rotationally invariant.

We have not added a small imaginary part to the self-energy above the light cone in the theory lines in the plots for the longitudinal propagator, but the data clearly show that one is present. The agreement between data and theory is generally good for both longitudinal and transverse propagators, and the data for the two values of $k$ disagree by about the same amount as the jitter in the data caused by statistical error. The comparison to the theory 

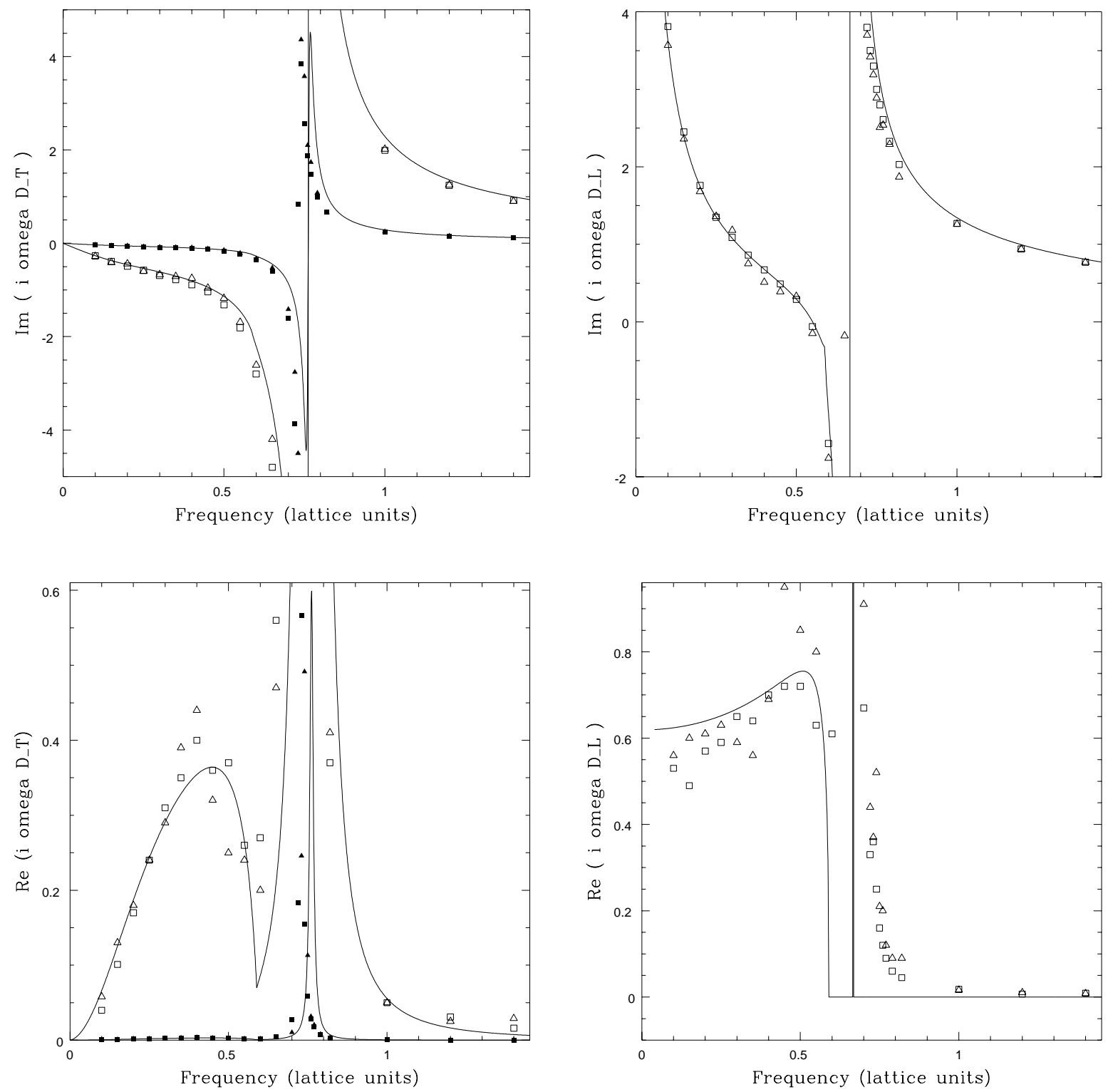

Figure 5: Retarded Propagator-actually $i \omega D^{\mathrm{R}}$. Above left, the out of phase (nondissipative) part of the transverse propagator; lower left, the in phase (dissipative) part of the transverse propagator. The right figures are the same for the longitudinal propagator. In each case, the solid lines are theory, the squares are the $k=(3,0,0) \times \pi / 16$ data, and the triangles are the $k=(2,1,1) \times \pi / 16$ data. In the transverse theory figures, the curves with smaller, filled data points are rescalings of the data and theory, so the resonance will fit in the plot; the rescalings are by 8 and 120 for the out of phase and in phase responses respectively. All numbers are in lattice units. 
without hard thermal loops is stark; for instance, the in phase response would then be zero in all cases, and the out of phase, longitudinal response would have no resonance but would behave simply as $1 / \omega$.

We conclude that the particles add hard thermal loops which, for $k \ll \pi$ in lattice units, are rotationally invariant and very close to the correct "hard thermal loops", except for corrections which are $O\left(\omega^{2}\right)$ and $O\left(Q^{4} \beta_{\mathrm{L}}^{2}\langle n\rangle\right)$ (both in lattice units). This is totally unlike the hard thermal loops induced by hard lattice modes in the nonabelian theory or the abelian Higgs model, which are rotationally non-invariant [20].

\section{Diffusion of Chern-Simons number}

An outstanding question which the method developed here can answer is; what is the diffusion constant for Chern-Simons number $N_{\mathrm{CS}}$ in the symmetric electroweak phase, or in pure Yang-Mills theory? In the continuum, the Chern-Simons number is defined as

$$
\begin{aligned}
\partial^{\mu} K_{\mu} & \equiv \frac{g^{2}}{32 \pi^{2}} \frac{\epsilon^{\mu \nu \alpha \beta}}{2} F_{\mu \nu}^{a} F_{\alpha \beta}^{a} \\
N_{\mathrm{CS}} \equiv \int d^{3} x K_{0} & =\frac{g^{2}}{32 \pi^{2}} \int d^{3} x \epsilon_{i j k}\left(F_{i j}^{a} A_{k}^{a}-\frac{g}{3} f_{a b c} A_{i}^{a} A_{j}^{b} A_{k}^{c}\right) .
\end{aligned}
$$

where Latin indices $(i j k)$ imply sums over the three space directions with positive metric. In vacuum $N_{\mathrm{CS}}$ is an integer, equal to the winding number of the gauge transformation which carries the gauge fields into the trivial fields $A=0$. This can be nonzero for groups with nontrivial third homotopy group. Chern-Simons number is of interest physically because of how it is related to the behavior of fermions coupled to the gauge fields; a vacuum to vacuum process which changes $N_{\mathrm{CS}}$ by $n$ pulls $n$ left handed negative energy solutions of the Dirac operator up to positive energy and pushes $n$ right handed solutions from positive to negative energy, if the fermion couples to the gauge fields in the fundamental representation. In a chiral theory, like the $\mathrm{SU}(2)$ sector of the standard model, no right handed particles couple, and there will be net particle creation (recall that in vacuum, negative energy solutions are occupied and positive energy solutions are not). Summing over the $N_{\mathrm{F}}=3$ generations one finds that the baryon number and lepton number currents both have nonzero divergences,

$$
\partial^{\mu} J_{\mu \mathrm{L}}=\partial^{\mu} J_{\mu \mathrm{B}}=N_{\mathrm{F}} \partial^{\mu} K_{\mu}
$$

so the number of baryons $N_{\mathrm{B}}$ and of leptons $N_{\mathrm{L}}$ changes as

$$
N_{\mathrm{B}}(t)-N_{\mathrm{B}}(0)=N_{\mathrm{L}}(t)-N_{\mathrm{L}}(0)=N_{\mathrm{F}}\left(N_{\mathrm{CS}}(t)-N_{\mathrm{CS}}(0)\right) .
$$

At high temperatures the efficiency with which $N_{\mathrm{B}}$ is violated is related to the diffusion constant for $N_{\mathrm{CS}}$,

$$
\Gamma \equiv \lim _{V \rightarrow \infty} \lim _{t \rightarrow \infty} \frac{\left\langle\left(N_{\mathrm{CS}}(t)-N_{\mathrm{CS}}(0)\right)^{2}\right\rangle}{V t}
$$


(where \langle\rangle refers to the thermal ensemble) by a fluctuation-dissipation relation [21, 44] and standard thermodynamic arguments [5]. In the minimal standard model[ one finds

$$
\frac{1}{N_{\mathrm{B}}+N_{\mathrm{L}}} \frac{d\left(N_{\mathrm{B}}+N_{\mathrm{L}}\right)}{d t}=\frac{39}{4 T^{3}} \Gamma .
$$

It would be phenomenologically interesting to know $\Gamma$ at high temperatures, which presumably existed in the early universe before the electroweak $\mathrm{SU}(2) \times \mathrm{U}(1)$ symmetry was spontaneously broken.

There has been a great deal of work to date on determining $\Gamma$ [17, 15, 22, 41, 42], but recently there have been two important developments.

The first is an analytic argument due to Arnold, Son, and Yaffe (ASY) [23]. They point out that a change in $N_{\mathrm{CS}}$ involves the evolution of very infrared magnetic fields. The hard thermal loops cause these fields to evolve in an overdamped manner. This is familiar from the study of abelian (electromagnetic) plasmas; magnetic fields of wavelength $\lambda \gg 1 / \omega_{\mathrm{p}}$ get "frozen" by the conductivity of the plasma and evolve on the time scale $\tau \sim \lambda\left(\lambda \omega_{\mathrm{p}}\right)^{2}$, assuming $\lambda$ is much shorter than the diffusion length of the charge carriers. ASY argue that the same physics should apply in the nonabelian plasma, at least for $\lambda$ less than or on order $1 / g^{2} T$; so $\Gamma$ should be parametrically of order

$$
\Gamma^{-1} \sim \lambda^{3} \tau \sim\left(\frac{1}{g^{2} T}\right)^{3} \frac{\omega_{\mathrm{p}}^{2}}{\left(g^{2} T\right)^{3}}
$$

If this argument is correct then $\Gamma$ depends strongly on the physics of hard thermal loops, which we must get right to find the correct $\Gamma$. Classical lattice theory by itself (without classical particles) does not. The damping coefficient describing the overdamped evolution of infrared magnetic fields on long time scales grows linearly with $1 / a$ and is not rotationally invariant 45.

The second development is that better definitions of $N_{\mathrm{CS}}$ on the lattice have been developed [25, 26, 46]. Previous definitions in terms of local operators contained lattice spacing dependent systematic errors. Moore and Turok [25] proposed a definition which is topological and hence avoids such errors. Their results verify that $\Gamma$ depends strongly on lattice spacing, in a manner which appears consistent with the ASY scaling law, though only if there are substantial corrections to that law at higher order in $\left(g^{2} T\right)^{2} / \omega_{\mathrm{p}}^{2}$.

By using the topological method of Moore and Turok to track the evolution of $N_{\mathrm{CS}}$, and by using the classical field theory with particles added to correctly reproduce the hard thermal loops, we can now get a determination of the diffusion constant for $N_{\mathrm{CS}}$ which accounts correctly both for topology and for hard thermal loops. We can also ensure that the thermodynamics of the system under study are correct by using the $O(a)$ improved matching developed in [11] and extended to arbitrary Debye mass in Appendix B.

We will do so in pure Yang-Mills theory, which should correspond to the very high temperature limit of the Standard Model because the thermal Higgs mass becomes large enough at high temperature that the Higgs field can be integrated out (though its contribution to

\footnotetext{
${ }^{6}$ In extensions with light baryon number or lepton number carrying particles, 39/4 will be replaced by something smaller; in the supersymmetric theory, if all the squarks and sleptons were light, 39/4 would become $39 / 12$.
} 
the hard thermal loops should of course be included). The sphaleron rate in the symmetric phase at the equilibrium point of the phase transition will differ somewhat from the YangMills theory value, in a way which depends on the (unknown) couplings of the Higgs sector. It is straightforward to add the Higgs field to the theory we have developed-in particular the influence of hard modes on the Higgs field can be completely accounted for by the choice of Higgs mass 47]-so there is no obstacle to extending what we do here to that case.

There is an important complication to our plan; in a nonabelian theory, the UV classical field modes will also generate hard thermal loops; and as Bödeker et. al. [20] have shown, the functional form of the hard thermal loops they provide is NOT the same as Eq. (11). Hence, the actual hard thermal loop contribution to the lattice system with particles will be of form

$$
m_{\mathrm{D}, \text { particles }}^{2} \cdot \Gamma_{\text {particles }}[A]+m_{\mathrm{D}, \mathrm{UV} \text { lattice }}^{2} \cdot \Gamma_{\mathrm{UV} \text { lattice }}[A],
$$

where $\Gamma_{\text {particles }}[A]$ has the correct and $\Gamma_{\text {UV lattice }}[A]$ has the wrong functional form. This is further complicated because of the interactions between the particles and the UV lattice modes. The UV lattice modes are Landau damped, and as we increase $m_{\mathrm{D}}^{2}$ from particles, that damping becomes stronger. If this damping is strong enough, then UV classical modes have short propagation distances, and they will not propagate interactions over large spatiotemporal separations. The hard thermal loop effects most important to the $N_{\mathrm{CS}}$ diffusion rate are those on length scales of order $1 / g^{2} T$ [23], so the relevant contribution from UV classical lattice modes may have an $m_{\mathrm{D}}^{2}$ dependent suppression. We do not know a good way to estimate the importance of this suppression, so we will treat it as a source of systematic error.

We want to know the result of a triple limit. The innermost limit is the limit of $Q^{2} \rightarrow 0$ and $\langle n\rangle \rightarrow \infty$ with $Q^{2}\langle n\rangle$ fixed; in this limit the particles generate only hard thermal loop

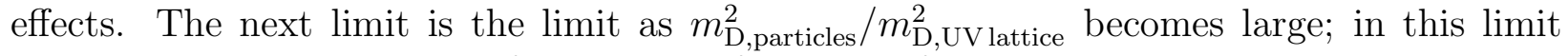
the hard thermal loops are of the correct functional form, plus a correction which is small relative to the total strength. At fixed lattice spacing, this limit means making $m_{\mathrm{D}}^{2}$ large; so we can only learn about the parametrically leading behavior in the limit of large $m_{\mathrm{D}}^{2}$. Finally, we should take a small lattice spacing limit.

Limited numerical resources make it impossible to really achieve this triple limit. We will choose a value for $Q^{2}$ small enough that we can expect to be in the relevant limit there, and we will also assume that $\beta_{L} \sim 10$ puts us far enough in the small $a$ limit, if $O(a)$ thermodynamic corrections are used]. We will check both of these limits by varying $Q^{2}$ holding $Q^{2}\langle n\rangle$ fixed and by varying $a$, to verify that the dependence is weak; but we make no serious attempt to extrapolate to these limits. We concentrate on what we consider the most phenomenologically interesting limit, what happens as we make $m_{\mathrm{D}}^{2}$ large. In particular we want to know whether the $\Gamma_{d}$ scales according to the ASY prediction, $\Gamma_{d} \propto m_{\mathrm{D}}^{-2}$.

We will then try to check three things:

1. $\Gamma$, in physical units, should depend weakly on the lattice spacing, provided that the physical value of the plasma frequency is held fixed;

\footnotetext{
${ }^{7}$ We verify in Appendix $\mathrm{A}$ that the frequency corrections to the particle hard thermal loops are $O\left(\omega^{2} a^{2}\right)$, and we believe the same should be true of the finite $k$ corrections. This leaves nonrenormalizable operators, also $O\left(k^{2} a^{2}\right)$, thermodynamic errors beyond $O(a)$, and an $O(a)$ rescaling of the time scale, which we estimate in Appendix D but have not computed.
} 


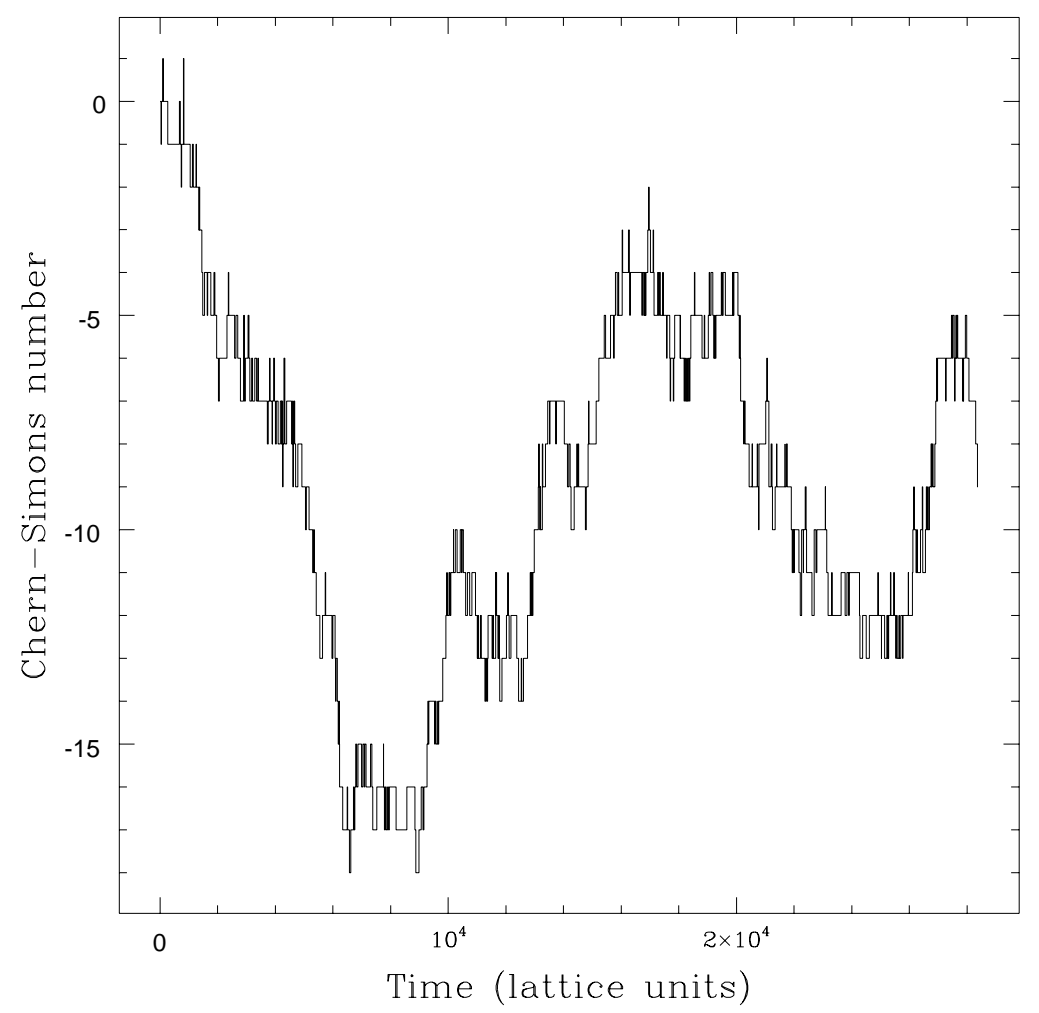

Figure 6: $\quad N_{\mathrm{CS}}$ during a Hamiltonian evolution, tracked by the slave field method (which returns the integer value of $N_{\mathrm{CS}}$ for the nearest vacuum). This is part of the data for row (5) of the table. The back and forth stuttering accompanying many winding number changes is an expected feature if the ASY picture is right 223.

2. $\Gamma$ should depend on $Q^{2}$ and $\langle n\rangle$ only through the combination $Q^{2}\langle n\rangle$, when we have chosen $Q^{2}$ small enough that the particle trajectories are ballistic on the nonperturbative scale $\left(g^{2} T\right)^{-1}$;

3. $\Gamma$ should depend inversely on hard thermal loop strength, up to corrections due to the UV classical lattice mode contributions.

To test 1., we measure $\Gamma$ at three lattice spacings, but with the same value of $Q^{2}$ and $\langle n\rangle$ in physical units. To test 2 ., we double the value of $\langle n\rangle$ and halve the value of $Q^{2}$ relative to the runs used in 1., and to test 3 . we double and quadruple $\langle n\rangle$ relative to the runs used to study 1., but keep the same value of $Q^{2}$. If the ASY scaling law is right, then up to corrections due to HTL's from UV classical lattice modes, all these runs should give the same value for $\Gamma$, except for the last, where $\Gamma$ should be about half and then a quarter as large. If $\Gamma$ does not depend on hard thermal loops, then all the results should be the same, and should be the same as the result without any particles added.

Our results are presented in Table 1. Each data point is extracted from several Hamiltonian evolutions from independent thermal initial conditions. We present a sample of $N_{\mathrm{CS}}$ during such a Hamiltonian trajectory in Figure 6. The analysis techniques used to extract $\Gamma$ are the same as in [25]. For each choice of parameters the sum of lengths of evolutions is about 90000 lattice units of time. The value of $\langle n\rangle$ for the $\beta_{\mathrm{L}}=8,10,12$ data keeps the 


\begin{tabular}{|c|c|c|c|c|c|}
\hline$\beta_{\mathrm{L}, \mathrm{imp}}$ (improved) & lattice size & $\langle n\rangle$ & $Q^{2}$ & $\kappa$ & $\kappa^{\prime}$ \\
\hline 8 & $20^{3}$ & 58.6 & .0064 & $.84 \pm .08$ & $49 \pm 5 \pm 7$ \\
\hline 10 & $24^{3}$ & 30.0 & .0064 & $.92 \pm .07$ & $56 \pm 4 \pm 10$ \\
\hline 12 & $30^{3}$ & 17.4 & .0064 & $.72 \pm .07$ & $46 \pm 5 \pm 10$ \\
\hline 10 & $24^{3}$ & 60.0 & .0032 & $.72 \pm .06$ & $44 \pm 4 \pm 8$ \\
\hline 10 & $24^{3}$ & 60.0 & .0064 & $.475 \pm .041$ & $53 \pm 5 \pm 5$ \\
\hline 10 & $24^{3}$ & 120.0 & .0064 & $.249 \pm .025$ & $53 \pm 5 \pm 2$ \\
\hline
\end{tabular}

Table 1: $\quad N_{\mathrm{CS}}$ diffusion constant in physical units, $\Gamma=\kappa \alpha^{4} T^{4}$, varying lattice spacing, particle charge, and particle number. The inverse lattice spacing $\beta_{\mathrm{L}}=4 / g^{2} a T$ used here is the one including the perturbative corrections found in 11] and Appendix B. The last column is the coefficient of the ASY scaling law, see the text.

physical particle density, which is proportional to $\beta_{\mathrm{L}}^{3}\langle n\rangle$, fixed; and as we have discussed, $Q^{2}$ does not scale with lattice spacing. Hence these three results test (1). Row 4 has the same value of $Q^{2}\langle n\rangle$ as row 2, so comparing them tests (2). Finally, comparing rows 2, 5, and 6 tests $(3)$.

The results are expressed through the dimensionless quantity $\kappa$, which in the continuum is defined through

$$
\Gamma=\kappa \alpha^{4} T^{4}
$$

or on the lattice,

$$
\Gamma=\kappa\left(\beta_{\mathrm{L}, \mathrm{imp}} \pi\right)^{-4}
$$

times a correction, discussed in Appendix D, to account for the correct matching of the time scales.

We also present the results in terms of the coefficient of the ASY scaling law, which we write as

$$
\Gamma=\kappa^{\prime}\left(\frac{g^{2} T^{2}}{m_{\mathrm{D}}^{2}}\right) \alpha^{5} T^{4} .
$$

We use $m_{\mathrm{D}}^{2}$ here because it most conveniently characterizes the size of hard thermal loop effects. For the particle degrees of freedom, the Debye mass in physical units is

$$
m_{\mathrm{D}}^{2}=\frac{Q^{2}\langle n\rangle(\text { latt. units }) \beta_{\mathrm{L}}^{3} g^{4} T^{2}}{48}
$$

which for the first 4 columns equals $4 g^{4} T^{2}$, a little less than the physical value, which is $11 g^{2} T^{2} / 6, g^{2} \simeq 0.4$ [8].

Really the ASY scaling law says the results should depend not on the Debye mass but on a damping coefficient proportional to the transverse self-energy at $\omega \ll k \sim g^{2} T$. This is simply related to $m_{\mathrm{D}}^{2}$ in the case that the hard particles have a rotationally invariant spectrum and move at the speed of light. The particle degrees of freedom satisfy this requirement, but the hard classical lattice modes do not, so we have accounted for their contribution using the techniques of Arnold [45]. His result is that the ratio of damping coefficient to Debye mass squared is roughly $(0.68 \pm 0.2)$ times smaller for hard classical lattice modes than for ultrarelativistic particles. However, Landau damping of the UV classical lattice modes, 
mentioned earlier, may suppress their contribution to the transverse self-energy at $k \sim g^{2} T$; since we do not know how to compute the extent of this suppression, there is a systematic error. The upper limit of the systematic error bar we present is if they contribute fully, in which case we add 0.68 times the Debye mass squared from classical lattice modes to that from particles when converting $\kappa$ to $\kappa^{\prime}$. (In physical units the Debye mass squared from classical lattice modes is $m_{\mathrm{D}}^{2}($ latt $)=\left(\Sigma \beta_{\mathrm{L}} / 4 \pi\right) g^{4} T^{2}$.) The lower limit is if their contribution is fully frustrated by Landau damping off of hard particles, in which case we just use $m_{\mathrm{D}}^{2}$ from particles to convert from $\kappa$ to $\kappa^{\prime}$. The larger $Q^{2}\langle n\rangle$, the stronger the Landau damping; so the systematic is not common to all runs.

Our results are roughly consistent with lattice spacing independence and with dependence on $Q^{2}$ and $\langle n\rangle$ only through the combination $Q^{2}\langle n\rangle$. There does seem to be a weak systematic trend in lattice spacing, which could be partly from $O\left(a^{2}\right)$ effects we have not attempted to compute. For instance, besides the $O(a)$ corrections to the thermodynamics computed in [11] and Appendix $\mathbb{B}$, there are two $O\left(a^{2}\right)$ corrections: a renormalization of the coupling, and a nonrenormalizable $\left(D_{i} F_{i j}\right)^{2}$ term, which appears in the Hamiltonian with a negative sign. Both would raise the rate on coarser lattices. There are also $O\left(a^{2}\right)$ corrections to the Wong's equation limit of the interactions between particles and long wavelength modes. There also may be a weak trend in $Q^{2}$ when $Q^{2}\langle n\rangle$ is held constant, because we are not sufficiently close to the small $Q^{2}$ and large $\langle n\rangle$ limit. Not being in this limit means that the eikonal approximation used to turn the particles into hard thermal loop effects is not quite true. Scattering of the particles will tend to reduce their effectiveness. Hence one might expect a weak systematic where $\kappa$ rises with $Q^{2}$ at $Q^{2}\langle n\rangle$ fixed. Row 4 in the table suggests this but the effect is not very statistically significant. Systematics have not been eliminated but they are small.

Our results rule out the "old picture" that $\Gamma$ should depend on $\alpha^{4} T^{4}$ by demonstrating the importance of hard thermal loop effects. If the $\alpha^{4} T^{4}$ law were correct, all the values for $\kappa$ would agree, and would agree with the value in Yang-Mills theory without particles, which is $\kappa=1.53 \pm 0.10$ at $\beta_{\mathrm{L}}=10$ [25]. The three results, rows (2), (5), and (6) of the table, differ only in the number of particles used, not in the lattice spacing, the particle charge, or the manner in which finite $\beta_{\mathrm{L}}$ lattice spacing systematics were taken care of. They are grossly in conflict if $\kappa$ is independent of $m_{\mathrm{D}}^{2}$, but they agree very nicely with the ASY scaling law. We illustrate this in Figure 7. Combining them, we get an estimate for $\kappa^{\prime}$ of $\kappa^{\prime}=53 \pm 3_{\text {stat }} \pm 5_{\text {syst }}$. We should also fold in the systematic errors from finite lattice spacing and finite $Q^{2}$, which we would estimate based on the other runs to be in the $20 \%$ range. These then dominate our uncertainties, and our final answer is $\kappa^{\prime}=53 \pm 11$. For comparison, we can take the results for pure Yang-Mills theory without particles from [25] and extrapolate them to zero lattice spacing assuming the ASY scaling applies. The extrapolation is $\kappa=23.6 / \beta_{\mathrm{L}}$, see figure 7 of that paper. We can convert this into a value for $\kappa^{\prime}$ by using the discussion after Eq. (74), that is, using Arnold's calculation of the relation between damping from hard classical lattice modes and from correct hard thermal loops [45]. We get $\kappa^{\prime}=51 \pm 15$.] The error here is almost all systematic, arising from the rotational noninvariance of the spectrum of lattice modes.

It is encouraging that these results agree within (admittedly substantial and mainly

\footnotetext{
${ }^{8}$ We thank Peter Arnold and Jan Smit for discussions on this point.
} 


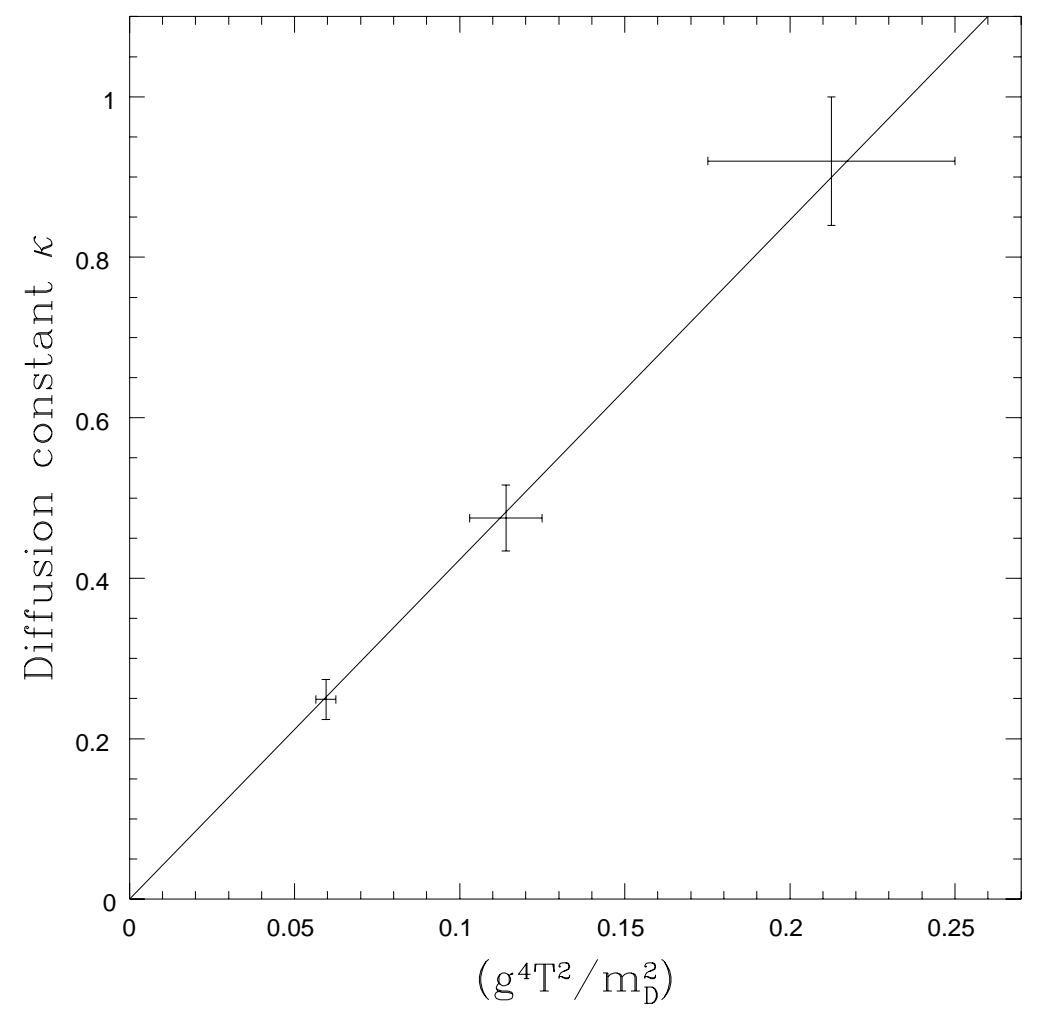

Figure 7: Results for $\kappa$ at three values of particle number but a common value of lattice spacing, plotted against $g^{4} T^{2} / m_{\mathrm{D}}^{2}$. The error bars in $m_{\mathrm{D}}^{2}$ reflect uncertainty in the damping from hard classical lattice modes. The "old picture" predicts a flat line, while the ASY picture predicts a straight line through the origin, like the illustrated fit.

systematic) errors. It appears that a consistent picture for the $N_{\mathrm{CS}}$ diffusion constant has emerged.

\section{Conclusions}

We have developed a procedure for generating the nonlocal "hard thermal loop" effects in classical field simulations of Yang-Mills theory, by introducing particle degrees of freedom which generate these effects but can be treated with reasonable numerical effort. The new system is Hamiltonian and has the same thermodynamics as thermal Yang-Mills theory in the dimensional reduction approximation. We have also tested that the self-energy corrections due to the particles in the abelian theory are correct, and we have analytically computed the plasma frequency at leading order and it is also correct.

Further, we have applied the technique to the calculation of the diffusion constant of Chern-Simons number in pure Yang-Mills theory. Our results vindicate the proposed scaling law of Arnold, Son, and Yaffe, that the diffusion constant $\Gamma$ should scale inversely with the square of the Debye mass. This statement is not sensitive to the quality of the thermodynamic corrections we have applied, because we have data with different numbers of particles which show starkly different diffusion rates, but the lattice spacing and hence the 
thermodynamic corrections we have applied are the same. Our results (in Yang-Mills theory, which will be valid in Yang-Mills Higgs theory only for thermal Higgs mass $m_{H}^{2}(T) \gg g^{4} T^{2}$ ) convert to $\Gamma=29 \pm 6 \alpha^{5} T^{4}$ at $m_{\mathrm{D}}^{2}=11 g^{2} T^{2} / 6$.

There are no obstacles to extending our techniques to Yang-Mills Higgs theory or groups larger than $\mathrm{SU}(2)$, which would allow us to determine the screening dependence of a number of other dynamical properties of interest for baryogenesis, such as the IR bosonic contribution to the bubble wall friction and the strong sphaleron rate. The technique may also have applications for relativistic heavy ion collisions.

\section{Acknowledgments}

We would like to acknowledge Peter Arnold, Dam Son, Misha Shaposhnikov, Keijo Kajantie, Jan Smit, Dirk Rischke, and Sergei Matinyan for useful conversations. GM is grateful to Duke University for hospitality during a brief visit there. This work was supported in part by the U.S. Department of Energy (Grant No. DE-FG02-96ER40495) and by the North Carolina Supercomputing Center and the Pittsburgh Computing Center.

\section{A Plasma frequency in the abelian theory}

We have shown that the thermodynamic modifications due to particles are very close to those expected, and we have argued that the role of the particles in generating hard thermal loops should be the same as in the continuum theory, for suitably infrared and slowly varying gauge fields. Here we will check this, and test its limits, by explicitly calculating the plasma frequency, the oscillation frequency of a spatially homogeneous electric field, in the abelian theory. The calculation should apply approximately to the nonabelian theory, in the regime where $\omega_{\mathrm{p}} \gg g^{2} T$, since in this case the theory looks approximately abelian on the relevant length scales. Away from this limit, the plasma frequency of the nonabelian theory is not well defined, since the electric fields only oscillate coherently on a time scale of order $1 / g^{2} T$. We will calculate in the formal small $Q$, large $\langle n\rangle$, but finite $Q^{2}\langle n\rangle$ limit, but we will deal explicitly with the discrete nature of the lattice electric fields and their interactions with particles.

First we will consider the case of a continuous electric field. It is sufficient to consider an electric field pointing along a lattice direction, since we will work to linear order in the field strength, so general fields can be studied as a linear combination. We take the field to be $E_{i}(t)=\delta_{i x} \operatorname{Re} E_{0} e^{i \omega t}$, with $E_{0}$ a constant giving the strength of the electric field, and we will solve self-consistently for $\omega$. Consider a particle of charge $q \ll 1$ propagating in the background of this field, with a mean momentum in the $x$ direction of $p_{x}$ and a momentum orthogonal to the $x$ direction of $p_{\perp}$. The $x$ momentum will have a time dependent disturbance of $\delta p_{x}(t) \propto q E_{0}$, due to the electric field, which satisfies

$$
\delta \dot{p}_{x}=q E_{x}=\operatorname{Re} q E_{0} e^{i \omega t} \rightarrow \delta p_{x}=\operatorname{Re} \frac{-i q E_{0}}{\omega} e^{i \omega t} .
$$

The electric field responds to a current in the $x$ direction due to this particle, of magnitude 
$q v_{x}$. Expanding in $\delta p_{x} \ll 1$, This is

$$
v_{x}=\frac{p_{x}+\delta p_{x}}{\sqrt{p_{\perp}^{2}+p_{x}^{2}+2 p_{x} \delta p_{x}}} \simeq \frac{p_{x}}{p}+\frac{\delta p_{x}}{p}\left(1-\frac{p_{x}^{2}}{p^{2}}\right)=\frac{p_{x}}{p}+\frac{\delta p_{x} p_{\perp}^{2}}{p^{3}} .
$$

Now averaging over particles, the leading term will cancel between particles of opposite sign for $q$, and the time derivative of the electric field will be

$$
-\dot{E}_{x}=\frac{1}{V} \sum_{\text {particles }} \frac{q \delta p_{x} p_{\perp}^{2}}{p^{3}}=\operatorname{Re} \frac{-i q^{2} E_{0}}{\omega} e^{i \omega t} \sum_{\text {particles }} \frac{p_{\perp}^{2}}{p^{3}} .
$$

Now $\dot{E}_{x}=\operatorname{Re} i \omega E_{0} e^{i \omega t}$. The $E_{0}$ cancel on the two sides, and we get an expression for $\omega^{2}$. A nice way of thinking of this is that what we want to know is $\operatorname{Re}(-i \dot{E} / E)=\omega$; we just plug the determined value of $\dot{E}$ into this expression to find $\omega$.

Now we evaluate the expression. The average over angles of $p_{\perp}^{2}$ is $2 p^{2} / 3$, and the average over particles of $1 / p$ is $1 / 2 T$.

$$
\omega^{2}=\frac{\langle n\rangle\left\langle q^{2}\right\rangle}{3 T} .
$$

In lattice units, $1 / T$ becomes $\beta_{\mathrm{L}}$. In the nonabelian case, $\left\langle q^{2}\right\rangle=Q^{2} / 3$, because that is the average of the square of the projection of a particle charge into one abelian subgroup. We therefore find $\omega_{\mathrm{p}}^{2}=m_{\mathrm{D}}^{2} / 3$, as it should.

Now let us include the discrete nature of the electric fields and their interactions with the particles. Again we take the electric field to be spatially uniform with the same value, and we will solve self-consistently for the plasma frequency.

Again, what we want to know is $\operatorname{Re}(-i \dot{E} / E)$, or its time average. We consider the influence of one particle, then we will average over all particles. Again the particle has a mean momentum in the $x$ direction of $p_{x}$ and a perpendicular momentum of $p_{\perp}$. We will often write \pm to mean $\operatorname{sign}\left(p_{x}\right)$ and $\mp$ to mean $-\operatorname{sign}\left(p_{x}\right)$.

The impulse on the electric field on some link, due to a particle crossing the dual lattice face the link penetrates, is of fixed modulus $\mp q$. Past interactions with $E$ change the current the particle induces only by changing the time at which the impulse is felt. 9 Let the particle passage time, at zero order in $q$, be $t_{\text {cross. }}$. This crossing time will receive a correction due to past interactions of the particle with the electric field, which we denote $\delta t\left(t_{\text {cross }}\right)$. The particle's contribution to $\operatorname{Re} \int d t(-i \dot{E} / E)$ is

$$
\begin{aligned}
E_{0} \operatorname{Re} \int d t(-i \dot{E} / E) & =\operatorname{Re}\left[( \pm i q) e^{-i \omega\left(t_{\text {cross }}+\delta t\left(t_{\text {cross }}\right)\right)}\right] \\
& =\operatorname{Re}\left[( \pm i q) e^{-i \omega t_{\text {cross }}}\right]+\operatorname{Re}\left[ \pm q \omega \delta t\left(t_{\text {cross }}\right) e^{-i \omega t_{\text {cross }}}\right]
\end{aligned}
$$

The leading term is $q$ odd, and cancels between particles of opposite charge. But $\delta t\left(t_{\text {cross }}\right)$ will be $q$ odd at leading order, and this term will contribute at $O\left(q^{2}\right)$.

\footnotetext{
${ }^{9}$ There will also be a small number of particles which would have induced a current on this link, but instead induce a current on its neighbor. But there are an equal number which induce a current on this link rather than its neighbor, and this cancels out.
} 
Let us compute $\delta t(t)$ to leading order in $q$. It is related to the difference in the particle's position from the $E=0$ case, $\delta x(t)$, by $\delta t(t)=-\delta x(t)|p| / p_{x}$. This in turn is given by an integral over the particle's past history of the difference in its velocity from the average value,

$$
\delta x(t)=\int_{-\infty}^{t} d t^{\prime} \delta v_{x}\left(t^{\prime}\right)=\int_{-\infty}^{t} d t^{\prime} \delta p_{x}\left(t^{\prime}\right) \frac{p_{\perp}^{2}}{p^{3}} .
$$

Now $\delta p_{x}\left(t^{\prime}\right)$ is a sum over past crossings of the kick to the particle at that crossing. The particle has undergone an infinite number of past crossings, one for each plane parallel the plane of the dual lattice face it is currently crossing. The time since it crossed the plane a distance $\mp m$ away in the $x$ direction is $\pm m|p| / p_{x}$, and the phase of the electric field at that time was $\exp \left(i \omega\left(t_{\text {cross }} \mp m|p| / p_{x}\right)\right)$, and the impulse it received was, at leading order in $q$, $\pm q E|p| / p_{x}$. Hence,

$$
\left.\delta x\left(t_{\text {cross }}\right)\right)=\operatorname{Re}\left(e^{i \omega t_{\text {cross }}} \sum_{m=1}^{\infty} \frac{m|p|}{ \pm p_{x}} \frac{q E_{0}|p|}{ \pm p_{x}} \frac{p_{\perp}^{2}}{p^{3}} e^{\mp i \omega m|p| / p_{x}}\right) .
$$

This sum is not absolutely convergent. So to make sense of the calculation, we must regulate it. We do this by assuming that, as well as fluctuating with the plasma frequency, the correlator of $E$ at two times has a slow exponential envelope, so $E_{x}$ in the past is not $\operatorname{Re} E_{0} e^{i \omega t}$ but $\operatorname{Re} E_{0} e^{(i \omega+\epsilon) t}$. Of course, we will take the $\epsilon \rightarrow 0$ limit at the end.

Thus, after regulating the sum, the kick from this particle crossing this boundary is

$$
\operatorname{Re} \int d t \frac{-i \dot{E}}{E}=\operatorname{Re} \sum_{m=1}^{\infty} \mp \frac{m q^{2} \omega p_{\perp}^{2}}{p_{x}^{3}} e^{\mp(i \omega+\epsilon) m|p| / p_{x}} .
$$

The frequency with which a particle of this momentum encounters a boundary in the $x$ direction is $\pm p_{x} / p$, so $\omega$ is

$$
\omega=\frac{1}{V} \sum_{\text {particles }} \operatorname{Re} \sum_{m=1}^{\infty}-\frac{m q^{2} \omega p_{\perp}^{2}}{p_{x}^{2}|p|} e^{\mp(i \omega+\epsilon) m|p| / p_{x}} .
$$

Now writing $x=p_{x} /|p|$, and performing the integral over $p^{2} d p$ and over the aximuthal angle, this reduces to

$$
\omega^{2}=\frac{\langle n\rangle\left\langle q^{2}\right\rangle \beta_{\mathrm{L}}}{3} \operatorname{Re} \lim _{\epsilon \rightarrow 0} \frac{3 \omega^{2}}{2} \int_{0}^{1} d x \frac{x^{2}-1}{x^{2}} \sum_{m=1}^{\infty} m e^{(-i \omega-\epsilon) m / x} .
$$

The sum over $m$ may now be performed,

$$
\sum_{m=1}^{\infty} m e^{(i \omega-\epsilon) m / x}=\frac{1}{4} \sinh ^{-2}\left(\frac{\epsilon+i \omega}{2 x}\right) .
$$

Making the substitution $y=1 / x$, the integral becomes

$$
\omega^{2}=\frac{\langle n\rangle\left\langle q^{2}\right\rangle \beta_{\mathrm{L}}}{3} \operatorname{Re} \lim _{\epsilon \rightarrow 0} \frac{3 \omega^{2}}{2} \int_{1}^{\infty} d y \frac{1-y^{2}}{4 y^{2}} \sinh ^{-2}\left(\frac{y(\epsilon+i \omega)}{2}\right) .
$$


For $\epsilon>0$ the integral is exponentially convergent, and we can rotate the contour to run in the negative imaginary direction:

$$
\omega^{2}=\frac{\langle n\rangle\left\langle q^{2}\right\rangle \beta_{\mathrm{L}}}{3} \operatorname{Re} \lim _{\epsilon \rightarrow 0} \frac{3 \omega^{2}}{2} \int_{0}^{\infty} i d y \frac{(y+i)^{2}+1}{4(y+i)^{2}} \sinh ^{-2}\left(\frac{(y+i)(\omega-i \epsilon)}{2}\right) .
$$

The integral is well behaved and we are free to take the $\epsilon \rightarrow 0$ limit. Taking the real part, after some algebra we obtain

$$
\begin{aligned}
\omega^{2} & =\frac{\langle n\rangle\left\langle q^{2}\right\rangle \beta_{\mathrm{L}}}{3} \times F\left(\omega^{2}\right) \\
F\left(\omega^{2}\right) & =\frac{3 \omega^{2}}{2} \int_{0}^{\infty} d y \frac{2 y(\cosh (\omega y) \cos (\omega)-1)+\left(y^{4}+3 y^{2}\right) \sinh (\omega y) \sin (\omega)}{2\left(1+y^{2}\right)^{2}(\cosh (\omega y)-\cos (\omega))^{2}} \\
& \simeq 1-\frac{\omega^{2}}{4}+\frac{\omega^{4}}{240}+\frac{\omega^{6}}{30240} \ldots
\end{aligned}
$$

Here, of course, $\omega$ is the plasma frequency in lattice units, ie $a \omega$ in physical units. We see that it receives a correction when $\omega \sim 1 / a$, but that for $\omega^{2} a^{2} \sim 1 / 2$ in lattice units, the correction is already quite small. There will also be $O\left(\langle n\rangle Q^{4}\right)$ corrections, probably including damping, but we have not been able to calculate these analytically.

\section{B Integrating out the $A_{0}$ field at arbitrary $m_{\mathrm{D}}$}

We would like to understand the relation between the thermodynamics of infrared magnetic fields in our lattice system and the thermodynamics of infrared magnetic fields in the full quantum theory, say in the dimensional reduction approximation. As we have seen, our system has thermodynamics described by a lattice gauge theory with an $A_{0}$ field, which has a Debye mass $m_{\mathrm{DL}}^{2}$ which depends on $\beta_{\mathrm{L}}$ and on $\langle n\rangle Q^{2}$, whereas the dimensional reduction approximation gives the continuum limit of this lattice theory, with some particular renormalized $m_{\mathrm{D}}^{2}$ determined by the particle content of the full quantum field theory. There are two complications here, the difference in behavior between lattice and continuum systems and the difference in Debye mass. The first has been dealt with in 11], for the system without the $A_{0}$ field and for the system with the $A_{0}$ field but in the approximation that $m_{\mathrm{DL}}^{2}$ is small in lattice units. In the theory with only classical lattice modes this approximation is parametrically justified, but with the inclusion of particles this is not necessarily the case.

To deal with finite $m_{\mathrm{D}}^{2}$, one first notes that $m_{\mathrm{D}}$ is large enough to make the $A_{0}$ field heavy, and since the theory is super-renormalizable one can integrate over such heavy fields at one loop and capture their dominant contributions to the infrared. We should perform this integration in each system, leaving us to compare pure Yang-Mills theory in 3-D, on the lattice and in the continuum. The matching between these has been studied in [11] up to corrections of order $\beta_{\mathrm{L}}^{-2}$, so here we only discuss the integration over the $A_{0}$ field.

Integrating over the $A_{0}$ field in the continuum theory was studied in [8, 9]. There is only one correction, a self-energy correction to the gauge fields which shifts the gauge coupling $g_{3}^{2} \equiv g^{2} T$ to

$$
\bar{g}_{3}^{2}=g_{3}^{2}\left(1-\frac{g_{3}^{2}}{24 \pi m_{\mathrm{D}}}\right) \text {. }
$$




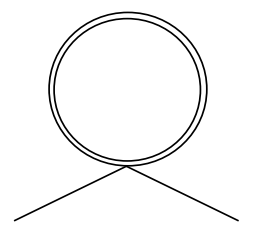

(a)

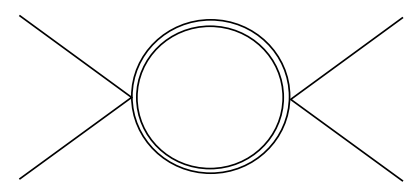

(b)

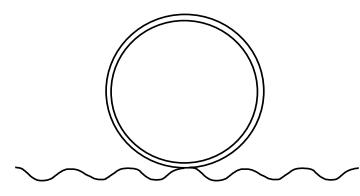

(c)

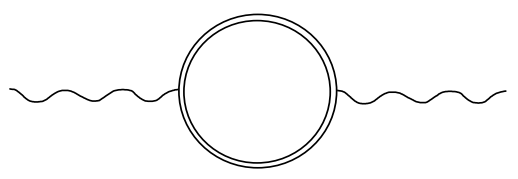

(d)

Figure 8: Diagrams needed in the integration over the $A_{0}$ field in Yang-Mills Higgs theory. The double solid line is the $A_{0}$ field, the single solid line is the Higgs field, and the wavy line is the gauge field.

In the theory with a Higgs field there is also a shift to the scalar self-coupling,

$$
\bar{\lambda}_{3}=\lambda_{3}-\frac{3 g_{3}^{4}}{128 \pi m_{\mathrm{D}}},
$$

and to the Higgs mass squared parameter,

$$
\bar{m}_{3}^{2}=m_{3}^{2}-\frac{3 g_{3}^{2} m_{\mathrm{D}}}{16 \pi}+O\left(g_{3}^{4} / 16 \pi^{2}\right) .
$$

The scalar wave function is not corrected.

In the lattice theory the integrals are trickier because the $A_{0}$ field has lattice dispersion relations and the gauge- $A_{0}$ vertices have nontrivial momentum dependence. So to warm up we will start with the corrections to the Higgs parameters. Our unit conventions will be the same as in [11], ie the lattice scalar self-coupling will be $\lambda_{\mathrm{L}}=4 \lambda / g^{2}$, and the lattice spacing will appear in the dimensionless quantity $\beta_{\mathrm{L}}=4 /\left(g^{2} a T\right)$.

Two diagrams are relevant, Fig. 8 diagrams $(a)$ and $(b)$. There are also high dimension operators induced by diagrams with more $\phi$ lines, but the influence of these terms is small for large $m_{\mathrm{D}}^{2}$ and we can drop them, just as in the continuum case [8]. Diagram (a) gives a momentum independent self-energy correction, ie a mass squared correction. Denote the coupling between the $A_{0}$ field and the Higgs field as $\lambda_{\mathrm{A}}$; at lowest order $\lambda_{\mathrm{AL}}=2$. Here and in what follows an $L$ subscript means the value in lattice units, with the normalizations used in [11. The shift in the lattice Higgs mass istu

$$
\bar{m}_{\mathrm{HL}}^{2}=m_{\mathrm{HL}}^{2}+\frac{3 h_{\mathrm{AL}}}{2 \beta_{\mathrm{L}}} \int_{[-\pi, \pi]^{3}} \frac{d^{3} k}{(2 \pi)^{3}} \frac{1}{\tilde{k}^{2}+m_{\mathrm{DL}}^{2}},
$$

where $\tilde{k}_{i} \equiv 2 \sin \left(k_{i} / 2\right)$ and

$$
\tilde{k}^{2}=\sum_{i} \tilde{k}_{i}^{2}=\sum_{i}\left(2-2 \cos k_{i}\right)
$$

Henceforth the range of integration and the $d^{3} k /(2 \pi)^{3}$ will be understood.

\footnotetext{
${ }^{10}$ Note that there are several typos in the Appendix $\mathrm{C}$ of [1] in which factors of $\Sigma / 4 \pi$ or $\xi / 4 \pi$ are left out.
} 
The integral in (93) must be determined numerically, and we define it as

$$
\int_{k} \frac{1}{\tilde{k}^{2}+m_{\mathrm{DL}}^{2}} \equiv \frac{\Sigma\left(m_{\mathrm{DL}}\right)}{4 \pi} \longrightarrow \frac{\Sigma}{4 \pi}-\frac{m_{\mathrm{DL}}}{4 \pi}-\frac{\xi m_{\mathrm{DL}}^{2}}{4 \pi}+\frac{m_{\mathrm{DL}}^{3}}{32 \pi}+\ldots
$$

where $\Sigma=\Sigma(0)=3.175911536$ and $\xi=0.152859325$. We display the small $m_{\mathrm{DL}}$ expansion, but at general $m_{\mathrm{DL}}$ the integral should be done numerically.

At zero external momentum diagram $(b)$ corrects the scalar self-coupling by

$$
\bar{\lambda}_{\mathrm{L}}=\lambda_{\mathrm{L}}-\frac{3 h_{\mathrm{AL}}^{2}}{4 \beta_{\mathrm{L}}} \int_{k} \frac{1}{\left(\tilde{k}^{2}+m_{\mathrm{DL}}^{2}\right)^{2}} .
$$

We define

$$
\int_{k} \frac{1}{\left(\tilde{k}^{2}+m_{\mathrm{DL}}^{2}\right)^{2}} \equiv \frac{\xi\left(m_{\mathrm{DL}}\right)}{4 \pi} \longrightarrow \frac{1}{8 \pi m_{\mathrm{DL}}}+\frac{\xi}{4 \pi}-\frac{3 m_{\mathrm{DL}}}{64 \pi}+\ldots
$$

Again, we have displayed the leading terms at small $m_{\mathrm{DL}}$, but at general $m_{\mathrm{DL}}$ the integral should be treated numerically. This definition of $\xi\left(m_{\mathrm{DL}}\right)$ does not have $\xi(m \rightarrow 0) \rightarrow \xi$; instead $\xi(m)$ has a pole at $m=0$, and $\xi$ is the constant part after the pole. The residue of the pole reproduces the continuum expression, (91), and the presence of the pole is because we are computing the full effect of the $A_{0}$ field on the lattice, not the difference between lattice and continuum theories.

Now we treat gauge field self-energy corrections. Since there are no $A_{0}$ field corrections to the three point gauge-scalar vertex or to the Higgs wave function at one loop, this is the only further correction we need. There are two diagrams, $(c)$ and $(d)$ of Figure 8. Denoting the polarization and spin indicies as $i, j$ and $a, b$, at general external momentum $p$, diagram $c$ gives

$$
-\frac{8 \delta_{a b} \delta_{i j}}{\beta_{\mathrm{L}}} \int \frac{\cos k_{i}}{\tilde{k}^{2}+m_{\mathrm{DL}}^{2}}
$$

and diagram $(d)$ gives

$$
\frac{\delta_{a b}}{\beta_{\mathrm{L}}} \int \frac{16 \sin k_{i} \sin k_{j}}{\left[(k-p / 2)^{2}+m_{\mathrm{DL}}^{2}\right]\left[(k+p / 2)^{2}+m_{\mathrm{DL}}^{2}\right]} .
$$

The sum of the contributions at $p=0$ is

$$
-\frac{\delta_{a b}}{\beta_{\mathrm{L}}} \int\left(\frac{8 \delta_{i j} \cos k_{i}}{\tilde{k}^{2}+m_{\mathrm{DL}}^{2}}-\frac{16 \sin k_{i} \sin k_{j}}{\left(\tilde{k}^{2}+m_{\mathrm{DL}}^{2}\right)^{2}}\right)=-4 \frac{\delta_{a b}}{\beta_{\mathrm{L}}} \int \frac{\partial^{2}}{\partial k_{i} \partial k_{j}} \ln \left(\tilde{k}^{2}+m_{\mathrm{DL}}^{2}\right)=0 .
$$

The last equality is because the integrand is a total derivative without singularities and the domain of integration is compact without boundary. Hence the $A_{0}$ field does not induce a mass for the gauge field, as ensured by gauge invariance.

Next, we find the $O\left(p^{2}\right)$ term in the self-energy, which is responsible for renormalizing the coupling. Only diagram $(d)$ depends on $p$, and expanding (99) to second order gives

$$
\frac{4 \delta_{A B}}{\beta_{\mathrm{L}}} \sum_{l m} p_{l} p_{m}\left(-\int \frac{2 \sin k_{i} \sin k_{j} \cos k_{l} \delta_{l m}}{\left(\tilde{k}^{2}+m_{\mathrm{DL}}^{2}\right)^{3}}+\int \frac{4 \sin k_{i} \sin k_{j} \sin k_{l} \sin k_{m}}{\left(\tilde{k}^{2}+m_{\mathrm{DL}}^{2}\right)^{4}}\right) .
$$


To evaluate this, we need the identity

$$
\begin{aligned}
0 & =\int \frac{\partial^{2}}{\partial k_{i} \partial k_{j}} \frac{\cos k_{l}}{\tilde{k}^{2}+m_{\mathrm{DL}}^{2}} \\
& =\int \frac{8 \sin k_{i} \sin k_{j} \cos k_{l}}{\left(\tilde{k}^{2}+m_{\mathrm{DL}}^{2}\right)^{3}}-\frac{2 \delta_{i j} \cos k_{i} \cos k_{l}}{\left(\tilde{k}^{2}+m_{\mathrm{DL}}^{2}\right)^{2}}+\frac{\cos k_{i} \delta_{i j l}}{\tilde{k}^{2}+m_{\mathrm{DL}}^{2}}
\end{aligned}
$$

where we have already used (100) to simplify the final expression, and the identity

$$
\begin{aligned}
0= & \int \frac{\partial^{4}}{\partial k_{i} \partial k_{j} \partial k_{l} \partial k_{m}} \ln \left(\tilde{k}^{2}+m_{\mathrm{DL}}^{2}\right) \\
= & -\int \frac{96 \sin k_{i} \sin k_{j} \sin k_{l} \sin k_{m}}{\left(\tilde{k}^{2}+m_{\mathrm{DL}}^{2}\right)^{4}}+\int 16 \frac{\delta_{i j} \cos k_{i} \sin k_{l} \sin k_{m}+5 \text { permutations }}{\left(\tilde{k}^{2}+m_{\mathrm{DL}}^{2}\right)^{3}}+ \\
& +\int \frac{6 \delta_{i j k l} \cos k_{i}}{\tilde{k}^{2}+m_{\mathrm{DL}}^{2}}-\int 4 \frac{\cos k_{i} \cos k_{l} \delta_{i j} \delta_{l m}+2 \text { permutations }}{\left(\tilde{k}^{2}+m_{\mathrm{DL}}^{2}\right)^{2}}
\end{aligned}
$$

Further, we use the relationship

$$
\sum_{i} \cos k_{i}=\left(3+\frac{m_{\mathrm{DL}}^{2}}{2}\right)-\frac{1}{2}\left(\tilde{k}^{2}+m_{\mathrm{DL}}^{2}\right),
$$

from which it follows that

$$
\int \frac{\cos k_{i}}{\tilde{k}^{2}+m_{\mathrm{DL}}^{2}}=-\frac{1}{6}+\left(1+\frac{m^{2}}{6}\right) \frac{\Sigma\left(m_{\mathrm{DL}}\right)}{4 \pi},
$$

and, with a little more work, that

$$
\begin{aligned}
\int \frac{\cos k_{i} \cos k_{j}}{\left(\tilde{k}^{2}+m_{\mathrm{DL}}^{2}\right)^{2}}= & {\left[\left(1+\frac{m_{\mathrm{DL}}^{2}}{2}+\frac{m_{\mathrm{DL}}^{4}}{24}\right) \frac{\xi\left(m_{\mathrm{DL}}\right)}{4 \pi}-\left(\frac{1}{4}+\frac{m_{\mathrm{DL}}^{2}}{24}\right) \frac{\Sigma\left(m_{\mathrm{DL}}\right)}{4 \pi}\right]+} \\
& +\delta_{i j}\left[-\left(\frac{m_{\mathrm{DL}}^{2}}{2}+\frac{m_{\mathrm{DL}}^{4}}{24}\right) \frac{\xi\left(m_{\mathrm{DL}}\right)}{4 \pi}-\left(\frac{1}{4}+\frac{m_{\mathrm{DL}}^{2}}{24}\right) \frac{\Sigma\left(m_{\mathrm{DL}}\right)}{4 \pi}+\frac{1}{12}\right]
\end{aligned}
$$

Using these, after considerable algebra we find that the $O\left(p^{2}\right)$ contribution to the self-energy is

$$
\frac{\delta_{a b}}{\beta_{\mathrm{L}}}\left(\delta_{i j} p^{2}-p_{i} p_{j}\right)\left[\left(-\frac{4}{3}-\frac{2 m_{\mathrm{DL}}^{2}}{3}-\frac{m_{\mathrm{DL}}^{4}}{18}\right) \frac{\xi\left(m_{\mathrm{DL}}\right)}{4 \pi}+\left(\frac{1}{3}+\frac{m_{\mathrm{DL}}^{2}}{18}\right) \frac{\Sigma\left(m_{\mathrm{DL}}\right)}{4 \pi}\right] .
$$

While intermediate expressions have been rotationally non-invariant, the result is rotationally invariant and transverse. This happens because the only gauge invariant dimension 4 operator which is cubic invariant is $F_{i j}^{2}$. At $O\left(p^{4}\right)$ there will be rotationally non-invariant contributions, although they will be cubic invariant. We are not concerned with these here because we are not trying to compute induced nonrenormalizable operators, only corrections to terms already in the Hamiltonian. But the nonrenormalizable terms could be computed by a straightforward but extremely tedious extension of what we have done here. 


\begin{tabular}{|c|c|c|c|c|c|}
\hline$\beta_{\mathrm{L}, \text { naive }}$ & $Q^{2}\langle n\rangle$ & $m_{\mathrm{D}}^{2}$ & $\Sigma\left(m_{\mathrm{D}}\right)$ & $\xi\left(m_{\mathrm{D}}\right)$ & $\beta_{\mathrm{L}, \mathrm{imp}}$ \\
\hline 8.7 & .375 & 1.59 & 1.901 & .3487 & 8.073 \\
\hline 10.7 & .189 & 1.09 & 2.101 & .4556 & 10.078 \\
\hline 12.7 & .111 & 0.74 & 2.280 & .5821 & 12.085 \\
\hline 10.7 & .378 & 1.77 & 1.840 & .3214 & 10.072 \\
\hline 10.7 & .756 & 3.14 & 1.498 & .1979 & 10.069 \\
\hline
\end{tabular}

Table 2: Conversion between $\beta_{\mathrm{L}, \text { naive }}$ and $\beta_{\mathrm{L} \text {,imp }}$ for the simulations in this paper. Everything is in lattice units. In Section 7 we truncate $\beta_{\mathrm{L}, \text { imp }}$ to the nearest integer when we write it.

The $m_{\mathrm{DL}}=0$ limit of ([107) agrees with the result in [1]], and the coefficient of the pole at $m_{\mathrm{DL}}=0$ reproduces (90). In the opposite limit of very large $m_{\mathrm{DL}}$ one can (Taylor) expand $\Sigma$ and $\xi$ in $m_{\mathrm{DL}}^{-2}$,

$$
\begin{aligned}
& \frac{\Sigma(m)}{4 \pi} \rightarrow m^{-2}-6 m^{-4}+42 m^{-6}-324 m^{-8}+2610 m^{-10}-\ldots \\
& \frac{\xi(m)}{4 \pi} \rightarrow m^{-4}-12 m^{-6}+126 m^{-8}-1296 m^{-10}+13050 m^{-12}-\ldots
\end{aligned}
$$

The expansion converges for $m^{2}>12=\sup \left(\tilde{k}^{2}\right)$. While the $\xi$ and $\Sigma$ contributions in (107) separately give $O\left(\mathrm{~m}^{0}\right)$ contributions, these cancel, as do the first several powers of $\mathrm{m}^{2}$, and the correction is $8 \delta_{a b}\left(p^{2} \delta_{i j}-p_{i} p_{j}\right) /\left(\beta_{\mathrm{L}} m_{\mathrm{DL}}^{8}\right)$ at leading order in $m_{\mathrm{DL}}^{-2}$. So the $A_{0}$ field rapidly becomes irrelevant at large Debye mass, as it should.

Combining the result from integrating out the $A_{0}$ field with the thermodynamic corrections from transverse gauge boson loops found in [11], we get a relation between the "naive" tree level $\beta_{\mathrm{L}, \text { naive }}$ and the improved value,

$\beta_{\mathrm{L}, \text { naive }}=\beta_{\mathrm{L}, \text { imp }}+\left(\frac{1}{3}+\frac{37 \xi}{6 \pi}\right)+\left[\left(-\frac{4}{3}-\frac{2 m_{\mathrm{DL}}^{2}}{3}-\frac{m_{\mathrm{DL}}^{4}}{18}\right) \frac{\xi\left(m_{\mathrm{DL}}\right)}{4 \pi}+\left(\frac{1}{3}+\frac{m_{\mathrm{DL}}^{2}}{18}\right) \frac{\Sigma\left(m_{\mathrm{DL}}\right)}{4 \pi}\right]$.

Here the constant $\xi=0.152859325$ is the limit of $\xi(m)$ as $m$ goes to zero, after the pole has been removed.

For completeness, we also list the values of $\beta_{\mathrm{L}, \text { imp }}$ and $\beta_{\mathrm{L}, \text { naive }}$ for the simulations presented in section 7, in Table 2.

This completes the integration over the $A_{0}$ field at one loop, at general $m_{\mathrm{DL}}^{2}$.

\section{Probing the retarded propagator by linear response}

In this appendix we show how to probe the retarded photon propagator by studying the linear response of the abelain plasma to an external current.

First we consider the response to transverse perturbations. In the framework of linear response theory, the response $A(x)$ is related to the external current $j(x)$ through the retarded propagator $D^{R}\left(x, x^{\prime}\right)$ :

$$
A_{i}(x)=\int d^{4} x^{\prime} D_{i k}^{R}\left(x, x^{\prime}\right) j_{k}\left(x^{\prime}\right)
$$


Going to Fourier space and suppressing the Lorentz indices, we have

$$
\tilde{E}(\omega, k)=i \omega \tilde{D}^{R}(\omega, k) \tilde{j}(\omega, k),
$$

where

$$
\tilde{D}^{R}(\omega, k)=\frac{-1}{\omega^{2}-k^{2}-\Pi(\omega, k)} .
$$

Taking $j_{i}(\vec{x}, t)=\hat{j}_{i} j_{0} e^{-i \omega_{0} t} e^{i k \cdot x} \theta(t)$ as the driving current, we find

$$
\tilde{E}_{i}(\omega, l)=\hat{j}_{i} \frac{j_{0} \omega(2 \pi)^{3} \delta^{3}(\vec{k}-\vec{l})}{\left(\omega-\omega_{0}+i \epsilon\right)\left(\omega^{2}-k^{2}-\Pi(\omega, k)\right)} .
$$

Fourier transforming $\tilde{E}(\omega, k)$ back to space-time gives

$$
E(\vec{x}, t)=j_{0} e^{i k_{0} x} \int \frac{d \omega}{2 \pi} \frac{\omega e^{-i \omega t}}{\left(\omega-\omega_{0}+i \epsilon\right)\left(\omega^{2}-k^{2}-\Pi(\omega, k)\right)} .
$$

The retarded propagator $D^{R}(\omega, k)$ is analytic in the upper half complex $\omega$-plane due to causality, and only has poles in the lower half plane. Writing $\Pi(\omega, k)=\Pi_{\mathrm{r}}(\omega, k)+i \Pi_{\mathrm{i}}(\omega, k)$ and noting that $\Pi_{\mathrm{r}}(-\omega, k)=\Pi_{\mathrm{r}}(\omega, k)$ and $\Pi_{\mathrm{i}}(-\omega, k)=-\Pi_{\mathrm{i}}(\omega, k)$, the two poles are located at $\omega_{\mathrm{pl}}\left(k_{0}\right)=\omega_{\mathrm{p}}\left(k_{0}\right)-i \gamma_{\mathrm{p}}\left(k_{0}\right)$ and $-\omega_{\mathrm{pl}}^{*}\left(k_{0}\right)$, where $\omega_{\mathrm{p}}\left(k_{0}\right)$ is the plasma frequency for the mode $k_{0}$ and $\gamma_{\mathrm{p}}\left(k_{0}\right)$ is the associated on-shell damping rate.

Completing the contour integral in the lower half $\omega$-plane, we find

$$
\begin{aligned}
E(\vec{x}, t)= & -i \frac{j_{0} \omega_{0} e^{-i \omega_{0} t} e^{i k_{0} x}}{\omega_{0}^{2}-k_{0}^{2}-\Pi\left(\omega_{0}, k_{0}\right)} \theta(t) \\
& -i \frac{j_{0} e^{-\gamma_{\mathrm{p}}\left(k_{0}\right) t} e^{i k_{0} x}}{\omega_{\mathrm{pl}}\left(k_{0}\right)+\omega_{\mathrm{pl}}^{*}\left(k_{0}\right)}\left[\frac{\omega_{\mathrm{pl}}\left(k_{0}\right) e^{-i \omega_{\mathrm{p}}\left(k_{0}\right) t}}{\omega_{\mathrm{pl}}\left(k_{0}\right)-\omega_{0}}-\frac{\omega_{\mathrm{pl}}^{*}\left(k_{0}\right) e^{i \omega_{\mathrm{p}}\left(k_{0}\right) t}}{\omega_{\mathrm{pl}}^{*}\left(k_{0}\right)+\omega_{0}}\right] \theta(t) .
\end{aligned}
$$

The first term represents the asymptotic response, while the second term is the plasma oscillation at $k=k_{0}$, which is only transient due to damping at a rate of $\gamma_{\mathrm{p}}\left(k_{0}\right)$. At large $t$ $\left(t \gg \gamma_{\mathrm{p}}^{-1}\left(k_{0}\right)\right)$, after the transients have died out, one sees only the asymptotic behavior

$$
E(\vec{x}, t) \longrightarrow-i \frac{j_{0} \omega_{0} e^{-i \omega_{0} t} e^{i k_{0} x}}{\omega_{0}^{2}-k_{0}^{2}-\Pi\left(\omega_{0}, k_{0}\right)} .
$$

A current of the form $j(\vec{x}, t)=j_{0} \sin \left(\omega_{0} t\right) \sin \left(k_{0} x\right) \cdot \theta(t)$ will generate the following $E$ field at large $t$,

$$
E(\vec{x}, t)=j_{0} \omega_{0} \rho \cos \left(\omega_{0} t+\alpha\right) \sin \left(k_{0} x\right) \cdot \theta(t)
$$

where

$$
\begin{aligned}
\rho & =\left\{\left[\omega_{0}^{2}-k_{0}^{2}-\Pi_{\mathrm{r}}\left(\omega_{0}, k_{0}\right)\right]^{2}+\Pi_{\mathrm{i}}^{2}\left(\omega_{0}, k_{0}\right)\right\}^{-\frac{1}{2}} \\
\alpha & =\tan ^{-1}\left[\frac{-\Pi_{\mathrm{i}}\left(\omega_{0}, k_{0}\right)}{\omega_{0}^{2}-k_{0}^{2}-\Pi_{\mathrm{r}}\left(\omega_{0}, k_{0}\right)}\right] .
\end{aligned}
$$


The real and imaginary part of $\Pi(\omega, k)$ can therefore be determined from the space-time averages

$$
\langle j(\vec{x}, t) \cdot E(\vec{x}, t)\rangle=\frac{j_{0}^{2}}{4} \cdot \frac{\omega_{0} \Pi_{\mathrm{i}}\left(\omega_{0}, k_{0}\right)}{\left[\omega_{0}^{2}-k_{0}^{2}-\Pi_{\mathrm{r}}\left(\omega_{0}, k_{0}\right)\right]^{2}+\Pi_{\mathrm{i}}^{2}\left(\omega_{0}, k_{0}\right)},
$$

and

$$
\langle j(\vec{x}, t) \cdot E(\vec{x}, t-\tau)\rangle=\frac{j_{0}^{2}}{4} \cdot \frac{\omega_{0}\left[\omega_{0}^{2}-k_{0}^{2}-\Pi_{\mathrm{r}}\left(\omega_{0}, k_{0}\right)\right]}{\left[\omega_{0}^{2}-k_{0}^{2}-\Pi_{\mathrm{r}}\left(\omega_{0}, k_{0}\right)\right]^{2}+\Pi_{\mathrm{i}}^{2}\left(\omega_{0}, k_{0}\right)},
$$

where $\tau=\pi /\left(2 \omega_{0}\right)$, and the averages are taken after the decay of the transients.

The numerically determined values for the transverse self-energies $\Pi_{i}$ and $\Pi_{r}$ can then be compared to their perturbative values 48]:

$$
\begin{aligned}
& \Pi_{\mathrm{r}}(\omega, k)=\frac{3}{2} \omega_{\mathrm{p}}^{2} \frac{\omega^{2}}{k^{2}}\left[1+\frac{1}{2}\left(\frac{k}{\omega}-\frac{\omega}{k}\right) \ln \left|\frac{\omega+k}{\omega-k}\right|\right], \\
& \Pi_{\mathrm{i}}(\omega, k)=-\frac{3 \pi}{4} \omega_{\mathrm{p}}^{2} \frac{\omega^{2}}{k^{2}}\left(\frac{k}{\omega}-\frac{\omega}{k}\right) \theta\left(k^{2}-\omega^{2}\right) .
\end{aligned}
$$

The response to longitudinal perturbations is easiest to treat in Coulomb gauge, where only $A_{0}$ is nonzero and $E$ is determined from $\partial_{i} A_{0}$. The retarded propagator is

$$
D_{00}^{\mathrm{R}}(\omega, k)=\frac{-\left(\omega^{2}-k^{2}\right)}{k^{2}\left(\omega^{2}-k^{2}-\Pi_{\mathrm{L}}(k, \omega)\right)} .
$$

One obtains

$$
\begin{aligned}
\langle j(x, t) \cdot E(x, t)\rangle & =\frac{j_{0}^{2}}{4 \omega} \frac{\left(\omega^{2}-k^{2}\right) \Pi_{\mathrm{L}, \mathrm{i}}}{\left(\omega^{2}-k^{2}-\Pi_{\mathrm{L}, \mathrm{r}}\right)^{2}+\Pi_{\mathrm{L}, \mathrm{i}}^{2}}, \\
\langle j(x, t) \cdot E(x, t-\tau)\rangle & =\frac{j_{0}^{2}}{4 \omega} \frac{\left(\omega^{2}-k^{2}\right)\left(\omega^{2}-k^{2}-\Pi_{\mathrm{L}, \mathrm{r}}\right)}{\left(\omega^{2}-k^{2}-\Pi_{\mathrm{L}, \mathrm{r}}\right)^{2}+\Pi_{\mathrm{L}, \mathrm{i}}^{2}} .
\end{aligned}
$$

The real and imaginary parts of the longitudinal self-energy are

$$
\begin{aligned}
& \Pi_{\mathrm{L}, \mathrm{r}}(\omega, k)=3 \omega_{\mathrm{p}}^{2}\left(1-\frac{\omega^{2}}{k^{2}}\right)\left(1-\frac{\omega}{2 k} \ln \left|\frac{\omega+k}{\omega-k}\right|\right), \\
& \Pi_{\mathrm{L}, \mathrm{i}}(\omega, k)=\frac{3 \pi}{2} \omega_{\mathrm{p}}^{2} \frac{\omega}{k}\left(1-\frac{\omega^{2}}{k^{2}}\right) \theta\left(k^{2}-\omega^{2}\right) .
\end{aligned}
$$

\section{Relating time scales when there are particles}

It has been shown [11], as discussed in Appendix B, that the difference in screening from UV modes between lattice gauge theory and continuum gauge theory can be understood at leading order in $a$ (or in $\beta_{\mathrm{L}}^{-1}$ ) as a rescaling of the differential operator $D_{i}$, so what at tree level looks like $D^{2} A$ in fact behaves like $Z_{A}^{-1} D^{2} A$, with $Z_{A}=1+O(a)$. The $O(a)$ term has been computed in 111 and extended to arbitrary Debye mass in Appendix B. Hence, in the case with no particles, the equations of motion for $A_{i}$ in temporal gauge look like

$$
\begin{aligned}
\frac{\partial E_{i}}{\partial t} & =-Z_{A}^{-1}\left(D^{2} \delta_{i j}-\frac{1}{2}\left(D_{i} D_{j}+D_{j} D_{i}\right)\right) A_{j}, \\
\frac{\partial A_{i}}{\partial t} & =Z_{E} E_{i},
\end{aligned}
$$


and $\left\langle E^{2}\right\rangle \propto \beta_{\mathrm{L}, \text { naive }}^{-1}$. The usual rule $\dot{A}=E$ is rescaled by $Z_{E}$, which has not been computed, but which lacks the large "tadpole" contributions which characterize $Z_{A}$. To get a complete $O(a)$ correction of the dynamics it would be necessary to compute $Z_{E}$, but it has been advocated in [25] that the absence of "tadpole" contributions means that $Z_{E}-1$ can be neglected compared to $Z_{A}-1$. In this case, a simple rescaling of time, $Z_{A}^{1 / 2} d / d t=d / d t^{\prime}$, and of the electric field, $Z_{A}^{1 / 2} E=E^{\prime}$, eliminates $Z_{A}$, and replaces $\beta_{\mathrm{L} \text {,naive }}$ with $\beta_{\mathrm{L}, \mathrm{imp}}=$ $Z_{A}^{-1} \beta_{\mathrm{L}, \text { naive }}$. So the time scale used, $t$, is related to the more appropriate time scale, $t^{\prime}$, by

$t^{\prime}=t \sqrt{\beta_{\mathrm{L}, \mathrm{imp}} / \beta_{\mathrm{L}, \text { naive }}}$. When one computes $\Gamma$ one should divide $\left\langle N_{\mathrm{CS}}^{2}\right\rangle$ by $V t^{\prime}$ rather than $V t$, and the corrected rate is $\sqrt{\beta_{\mathrm{L}, \text { naive }} / \beta_{\mathrm{L}, \mathrm{imp}}}$ times larger.

However we are now interested in the case where there are enough particles to put the evolution in the overdamped regime. Huet and Son [24] and Son 49] have recently shown what new term this adds to (130). A thermal distribution of particles would not contribute, since particles of opposite charge would cancel, but the particle distribution at any point is skewed because of past, remote electric fields, and contains fluctuations. The new evolution equation looks like

$$
\begin{aligned}
\frac{\partial E_{i}^{a}(x, t)}{\partial t}+Z_{A}^{-1} & \left(D^{2} \delta_{i j}-\frac{1}{2}\left(D_{i} D_{j}+D_{j} D_{i}\right)\right) A_{j}^{a}(x, t)=\frac{Q^{2}\langle n\rangle \beta_{\mathrm{L}, \text { naive }}}{3} \\
& \times \int d y N_{i j}^{a b}(x, y, t) E_{j}^{b}(y, t-|x-y|)+\text { (noise term) } .
\end{aligned}
$$

Here the right hand side represents the departure of the particle population from rotational invariance, due to its linear response to past electric fields, and fluctuations in the particle population. The form of the nonlocal kernel $N$ is given in [24, 49]. They point out that the overdamped limit corresponds to the large $Q^{2}\langle n\rangle$ limit, in which case the $\partial E / \partial t$ term and the time dependence of $E$ on the right hand side can be neglected. Again approximating that $\dot{A}=E$, rescaling time to eliminate $Z_{A}$ and to replace all appearances of $\beta_{\mathrm{L} \text {,naive }}$ with $\beta_{\mathrm{L}, \mathrm{imp}}$ now requires $t^{\prime}=Z_{A}^{-2} t$. Hence one should compute everything using $\beta_{\mathrm{L} \text {,imp }}$ and the lattice spacetime volume used, but then correct the rate by a factor of $\left(\beta_{\mathrm{L}, \text { naive }} / \beta_{\mathrm{L}, \mathrm{imp}}\right)^{2}$.

\section{References}

[1] A. Sakharov, JETP Lett. 6, 24 (1967).

[2] G. t'Hooft, Phys. Rev. Lett. 37,8 (1976).

[3] F. Klinkhamer and N. Manton, Phys. Rev. D 30, 2212 (1984).

[4] V. Kuzmin, V. Rubakov, and M. Shaposhnikov, Phys. Lett. D 30, 36 (1985).

[5] P. Arnold and L. McLerran, Phys. Rev. D 36, 581 (1987).

[6] P. Ginsparg, Nucl. Phys. B 170, 388 (1980); T. Applequist and R. Pisarski, Phys. Rev. D 23, 2305 (1981); S. Nadkarni, Phys. Rev. D 27, 917 (1983); N. P. Landsmann, Nucl. Phys. B 322, 498 (1989). 
[7] K. Farakos, K. Kajantie, K. Rummukainen, and M. Shaposhnikov, Nucl. Phys. B 425, 67 (1994).

[8] K. Kajantie, M. Laine, K. Rummukainen, and M. Shaposhnikov, Nucl. Phys. B 458, 90 (1996).

[9] K. Farakos, K. Kajantie, K. Rummukainen, and M. Shaposhnikov, Nucl. Phys. B 442, 317 (1995).

[10] M. Laine, Nucl. Phys. B 451, 484 (1995).

[11] G. D. Moore, Nucl. Phys. B 493, 439 (1997).

[12] K. Kajantie, M. Laine, K. Rummukainen, and M. Shaposhnikov, Nucl. Phys. B 466, 189 (1996); Phys. Rev. Lett. 77, 2887 (1996).

[13] H. Dosch, J. Kripfganz, A. Laser, and M. Schmidt, Phys. Lett. B 365, 213 (1996); M. Gurtler, E. Ilgenfritz, J. Kripfganz, H. Perlt, and A. Schiller, Nucl. Phys. B 483, 383 (1997); M. Gurtler, E. Ilgenfritz, and A. Schiller, Eur. Phys. J. C 1, 363 (1998); Phys. Rev. D 56, 3888 (1997).

[14] O. Philipsen, M. Teper, and H. Wittig, Nucl. Phys. B 469, 445 (1996); A. Hart, O. Philipsen, J. Stack, and M. Teper, Phys. Lett. B 396, 217 (1997).

[15] G. D. Moore and N. Turok, Phys. Rev. D 55, 6538 (1997).

[16] K. Kajantie, M. Laine, K. Rummukainen, and M. Shaposhnikov, Nucl. Phys. B 493, 413 (1997).

[17] J. Ambjørn and A. Krasnitz, Phys. Lett. B 362, 97 (1995).

[18] D. Grigorev and V. Rubakov, Nucl. Phys. B 299, 248 (1988).

[19] D. Bödeker, Nucl. Phys. B 486, 500 (1997).

[20] D. Bödeker, L. McLerran, and A. Smilga, Phys. Rev. D 52, 4675 (1995).

[21] S. Khlebnikov and M. Shaposhnikov, Nucl. Phys. B308, 885 (1988).

[22] W. Tang and J. Smit, Nucl. Phys. B 482, 265 (1996).

[23] P. Arnold, D. Son, and L. Yaffe, Phys. Rev. D 55, 6264 (1997).

[24] P. Huet and D. Son, Phys. Lett. B393, 94 (1997).

[25] G. D. Moore and N. Turok, Phys. Rev. D 56, 6533 (1997).

[26] J. Ambjørn and A. Krasnitz, Nucl. Phys. B 506, 387 (1997).

[27] K. Geiger, Phys. Rep. 258, 237 (1995).

[28] X. N. Wang, Phys. Rep. 280, 287 (1997). 
[29] M. H. Thoma, in Quark-Gluon Plasma 2, edited by R. C. Hwa, (World Scientific, Singapore, 1995), P. 51.

[30] C. Hu and B. Müller, Phys. Lett. B409, 377 (1997).

[31] P.F. Kelly, Q. Liu, C. Lucchesi, and C. Manuel, Phys. Rev. Lett. 72, 3461 (1994); Phys. Rev. D 50, 4209 (1994).

[32] E. Braaten and R.D. Pisarski, Nucl. Phys. B 337, 569 (1990).

[33] E. Braaten and R.D. Pisarski, Phys. Rev. D 42, 2156 (1990).

[34] J.C. Taylor and S.M.H. Wong, Nucl. Phys. B 346, 115 (1990).

[35] J.P. Blaizot and E. Iancu, Phys. Rev. Lett. 70, 3376 (1993).

[36] H. Elze and U. Heinz, Phys. Rep. 183, 81 (1989).

[37] S.K. Wong, Nuo. Cim. A 65, 689 (1970).

[38] U. Heinz, Phys. Lett. B 144, 228 (1984).

[39] R. Efraty and V.P. Nair, Phys. Rev. Lett. 68, 2891 (1992).

[40] J. Kogut and L. Susskind, Phys. Rev. D 11, 395 (1975).

[41] J. Ambjørn, T. Askgaard, H. Porter, and M. Shaposhnikov, Nucl. Phys. B 353, 346 (1991).

[42] G. D. Moore, Nucl. Phys. B 480, 657 (1996); Nucl. Phys. B 480, 689 (1996).

[43] A. Krasnitz, Nucl. Phys. B 455, 320 (1995).

[44] V. Rubakov and M. Shaposhnikov, Phys. Usp. 39, 461 (1996) [Usp. Fiz. Nauk 166, 493 (1996)].

[45] P. Arnold, Phys. Rev. D 55, 7781 (1997).

[46] G. D. Moore, Phys. Lett. B 412, 359 (1997).

[47] G. Aarts and J. Smit, Phys. Lett. B 393, 395 (1997).

[48] V. Klimov, Sov. J. Nucl. Phys. 33, 934 (1981); A. Weldon, Phys. Rev. D 26, 1394 (1982).

[49] D. Son, UW/PT-97-19, hep-ph/9707351. 